\title{
The OmegaWhite Survey for short period variable stars II: An overview of results from the first four years
}

\author{
R. Toma ${ }^{1 \star}$, G. Ramsay ${ }^{1}$, S. Macfarlane ${ }^{2,3}$, P. J Groot ${ }^{2}$, P. A. Woudt ${ }^{3}$, V. Dhillon ${ }^{4,6}$, \\ C. S. Jeffery ${ }^{1}$, T. Marsh ${ }^{5}$, G. Nelemans ${ }^{2}$, D. Steeghs ${ }^{5}$ \\ ${ }^{1}$ Armagh Observatory, College Hill, Armagh, BT61 9DG, UK \\ ${ }^{2}$ Department of Astrophysics/IMAPP, Radboud University, P.O. Box 9010, 6500 GL Nijmegen, The Netherlands \\ ${ }^{3}$ Department of Astronomy, University of Cape Town, Private Bag X3, Rondebosch 7700, South Africa \\ ${ }^{4}$ Department of Physics \& Astronomy, University of Sheffield, Sheffield S3 7RH, UK \\ ${ }^{5}$ Department of Physics, University of Warwick, Coventry CV4 7AL, UK \\ ${ }^{6}$ Instituto de Astrofisica de Canarias, E-38205 La Laguna, Tenerife, Spain
}

Accepted 2016 August 16. Received 2016 August 16; in original form 2016 July 15

\begin{abstract}
OmegaWhite is a wide-field, high cadence, synoptic survey targeting fields in the southern Galactic plane, with the aim of discovering short period variable stars. Our strategy is to take a series of $39 \mathrm{~s}$ exposures in the $g$ band of a 1 square degree of sky lasting $2 \mathrm{~h}$ using the OmegaCAM wide field imager on the VLT Survey Telescope (VST). We give an overview of the initial 4 years of data which covers 134 square degrees and includes 12.3 million light curves. As the fields overlap with the VLT Survey Telescope $\mathrm{H} \alpha$ Photometric Survey of the Galactic plane and Bulge (VPHAS+), we currently have ugri $H \alpha$ photometry for $\sim 1 / 3$ of our fields. We find that a significant fraction of the light curves have been affected by the diffraction spikes of bright stars sweeping across stars within a few dozen of pixels over the two hour observing time interval due to the alt-az nature of the VST. We select candidate variable stars using a variety of variability statistics, followed by a manual verification stage. We present samples of several classes of short period variables, including: an ultra compact binary, a DQ white dwarf, a compact object with evidence of a 100 min rotation period, three CVs, one eclipsing binary with an 85 min period, a symbiotic binary which shows evidence of a $31 \mathrm{~min}$ photometric period, and a large sample of candidate $\delta$ Sct type stars including one with a 9.3 min period. Our overall goal is to cover 400 square degrees, and this study indicates we will find many more interesting short period variable stars as a result.
\end{abstract}

Key words: surveys - binaries: close - Galaxy:bulge - methods: observational methods: data analysis - techniques: photometric.

\section{INTRODUCTION}

The aim of the OmegaWhite (OW) survey is to discover a population of short period variable stars close to the Galactic plane (Macfarlane et al. 2015, Paper I). In particular, our main goal is to discover ultra compact binaries (UCBs) which have orbital periods shorter than $70 \mathrm{~min}$. UCBs can take various guises including: X-ray binaries containing white dwarf - neutron star components (see e.g. Nelemans \& Jonker 2010), sub-dwarf B star - white dwarf components (e.g. Geier et al. 2013), double degenerate noninteracting binaries (e.g. Brown et al. 2011, 2016, Gianninas

^ Email: rto@arm.ac.uk et al. 2015), and the double degenerate interacting binaries (the AM CVn stars, see Solheim 2010 for a review).

UCBs are predicted to be strong persistent sources of low-frequency gravitational waves accessible with facilities such as the Evolved Laser Interferometer Space Antenna (eLISA, e.g. Nelemans 2013). Early estimates of the intrinsic number of AM CVn binaries suggested a relatively high gravitational wave background. However, in a series of papers using Sloan Digital Sky Survey (SDSS) data (York et al. 2000) it was established that the observed space density of AM CVn stars is $5 \pm 3 \times 10^{-7} \mathrm{pc}^{-3}$ (Carter et al. 2013): a factor of 12 lower than the 'pessimistic' model predictions outlined in Roelofs, Nelemans \& Groot 2007 which were based on the models of Nelemans et al. (2001, 2004). 
Table 1. The log for OW observations made during ESO Semesters 88-94. We show the calendar date, the number of fields which were observed and the range in their sky co-ordinates, and the number of light curves (prior to the flagging stage) which were obtained in each Semester.

\begin{tabular}{cccccc}
\hline $\begin{array}{c}\text { ESO } \\
\text { Period }\end{array}$ & Date & $\begin{array}{c}\text { Observed } \\
\text { Fields }\end{array}$ & $\begin{array}{c}\text { RA } \\
(\mathrm{J} 2000) \\
(\mathrm{hh}: \mathrm{mm})\end{array}$ & $\begin{array}{c}\text { DEC } \\
(\mathrm{J} 2000) \\
\left(:^{\prime}\right)\end{array}$ & $\begin{array}{c}\text { Number of } \\
\text { light curves } \\
\left(\times 10^{6}\right)\end{array}$ \\
\hline 88 & Dec 2011 $\rightarrow$ Apr 2012 & 26 & $07 \mathrm{~h} 35 \mathrm{~m} \rightarrow 08 \mathrm{~h} 25 \mathrm{~m}$ & $-30^{\circ} 00^{\prime} \rightarrow-26^{\circ} 00^{\prime}$ & 1.7 \\
90 & Nov 2012 $\rightarrow$ Mar 2013 & 4 & $07 \mathrm{~h} 05 \mathrm{~m} \rightarrow 08 \mathrm{~h} 25 \mathrm{~m}$ & $-30^{\circ} 00^{\prime} \rightarrow-25^{\circ} 00^{\prime}$ & 0.2 \\
91 & Apr 2013 $\rightarrow$ Sep 2013 & 26 & $17 \mathrm{~h} 00 \mathrm{~m} \rightarrow 18 \mathrm{~h} 25 \mathrm{~m}$ & $-29^{\circ} 30^{\prime} \rightarrow-23^{\circ} 30^{\prime}$ & 3.5 \\
92 & Dec 2013 $\rightarrow$ Apr 2014 & 8 & $07 \mathrm{~h} 05 \mathrm{~m} \rightarrow 08 \mathrm{~h} 40 \mathrm{~m}$ & $-31^{\circ} 00^{\prime} \rightarrow-26^{\circ} 00^{\prime}$ & 0.3 \\
93 & Apr 2014 $\rightarrow$ Sep 2014 & 34 & $17 \mathrm{~h} 05 \mathrm{~m} \rightarrow 18 \mathrm{~h} 30 \mathrm{~m}$ & $-32^{\circ} 30^{\prime} \rightarrow-21^{\circ} 30^{\prime}$ & 4.5 \\
94 & Dec 2015 $\rightarrow$ Apr 2015 & 36 & $07 \mathrm{~h} 15 \mathrm{~m} \rightarrow 08 \mathrm{~h} 30 \mathrm{~m}$ & $-32^{\circ} 00^{\prime} \rightarrow-23^{\circ} 00^{\prime}$ & 2.1 \\
\hline
\end{tabular}

The SDSS work led to the discovery of AM CVn stars with orbital periods in the range $35 \mathrm{~min}<P_{\text {orb }}<65 \mathrm{~min}$ (e.g. Roelofs et al. 2005, Anderson et al. 2005, 2008, Carter et al. 2014a, 2014b). More recently, the Palomar Transient Factory (PTF) survey has identified outbursting AM CVn binaries in the 20-35 minute period range (Levitan et al. 2011, 2013, 2014). It is systems with the shortest orbital period $(<20$ min) which are predicted to be the strongest emitters of gravitational waves. Determining their number is important for the development of eLISA and for understanding the relative importance of the three predicted formation channels of these binaries. One way to identify those AM CVn stars with the shortest orbital period is through their photometric behaviour as they often show a modulation on a period at, or close to, the orbital period (e.g. HM Cnc, Roelofs et al. 2010; AM CVn itself, Nelemans, Steeghs \& Groot 2001; SDSS J190817+3940, Kupfer et al. 2015).

The OW survey has a similar strategy to the RApid Temporal Survey (RATS, Ramsay \& Hakala 2005, Barclay et al. 2011, Ramsay et al. 2014). However, in contrast to the initial RATS survey, which had a sky coverage of 40 square degrees, OW has a goal of 400 square degree coverage. With a typical cadence of $3.6 \mathrm{~min}$, OW has a much higher cadence than the vast majority of wide field synoptic surveys. For instance, the PTF typically has a 5 day cadence (Law et al. 2009), and in the southern hemisphere, Skymapper (Keller et al. 2007) has a cadence of hours to years. Kepler/K2 is able to provide 1 min cadence but only for a small number of targets at any time (Gilliland et al. 2010). Additionally, unlike OW, most surveys exclude the Galactic Plane because of the high stellar density.

High cadence observations of fields along the Galactic plane will also deliver a comprehensive census of short period variable stars in the Galactic plane. These range from pulsating white dwarfs, sdB stars, $\delta$ Sct stars and their cousins the SX Phe and $\gamma$ Dor stars, as well as longer period systems such as contact binaries and transient phenomena such as flare stars.

In this paper, we outline the initial results of the first 4 years (2011 December to 2015 April) of the OW survey. We briefly summarise the reduction strategy, but highlight specific issues such as the process of flagging light curves which are likely to show spurious variability. We show some examples of new variables which are either examples of rare variable stars or can illuminate details of physical processes at work. In a companion paper (Macfarlane et al. 2016, Paper III) we present followup photometric and spectroscopic observations of variable stars identified in the OW survey which were made at the South African Astronomical Observatory, Sutherland, South Africa.

\section{OBSERVATIONS}

\subsection{Overview}

Observations are made using the 2.65-m VLT Survey Telescope (VST, Capaccioli \& Schipani 2011, Schipani et al. 2012) located at ESO's Paranal Observatory in Chile, and the OmegaCAM mosaic imager instrument (Kuijken 2011, Mieske et al. 2013) which has a field of view of one square degree which is covered by 32 CCDs with a pixel scale of $0.22 \mathrm{arcsec} / \mathrm{pixel}$. We take a sequence consisting of one 39 $\mathrm{s}$ exposure; move the telescope to an adjacent field; take another $39 \mathrm{~s}$ exposure; return to the first field and repeat for $2 \mathrm{~h}$ in total (all images are taken in the $g$ band). The mean cadence of images obtained in the Semesters covered in this paper is $3.6 \pm 0.7 \mathrm{~min}$, with a mean minimum cadence of $2.8 \mathrm{~min}$, where variations in the mean cadence are likely due to differences in the time it takes to do focussing and mirror alignment over the $2 \mathrm{~h}$ observation. In Paper I we presented the results of data obtained during Semester 88 . In this paper we present an overview of the data obtained during Semesters 88 and 90-94. For reference, we show in Table 1 the number of fields observed and in Figure 1 the sky position of these fields. In Table A1 we give details of the individual fields which were observed during Semester 90-94 (Paper I outlines the fields observed in Semester 88). Combined, these data cover 134 square degrees of sky and we obtain $12.3 \times 10^{6}$ light curves.

\subsection{Data Reduction}

Paper I presents details of our data reduction process which includes the image cleaning process, the photometric reduction, how we flag light curves which may have been contaminated by instrumental effects, and how we identify objects which are variable. For brevity, we only give a summary of these steps and the reader is directed to Paper I for more details of the individual stages. We highlight issues which may cause a degradation of the light curves.

(i) Data are downloaded from the ESO archive and the images are cleaned using a set of master bias images which have been made on the same day as the science images and 


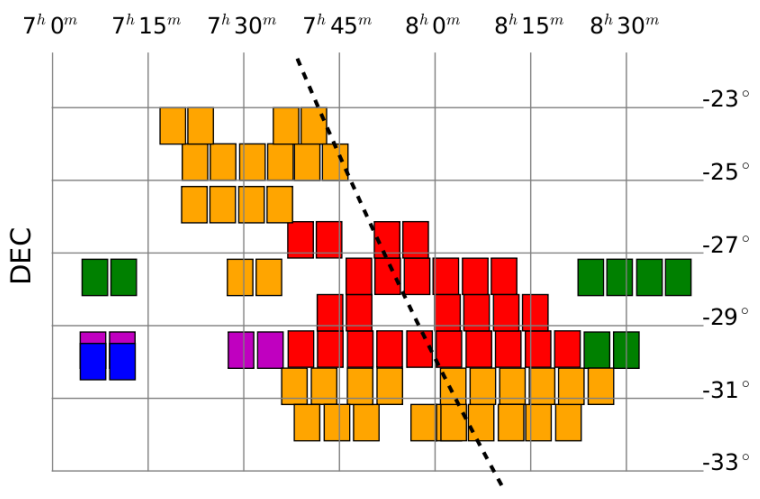

RA

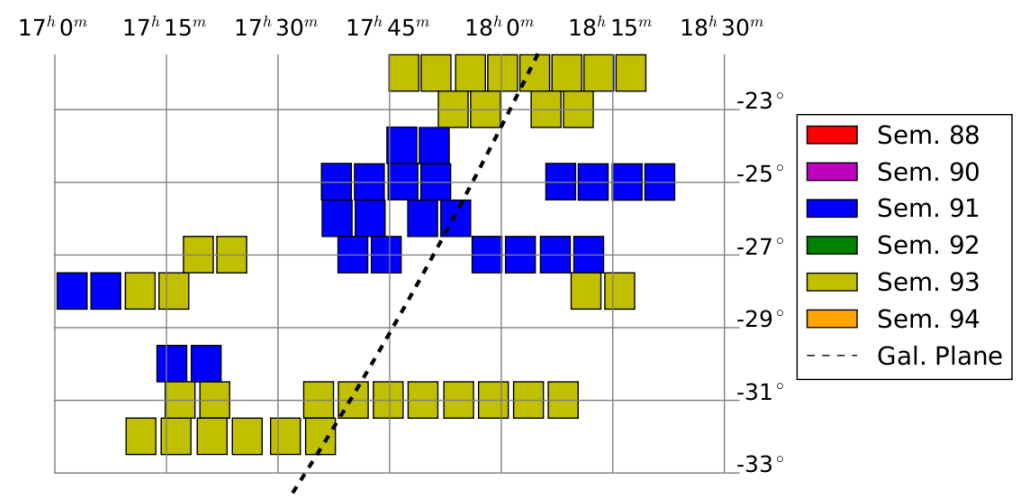

RA

Figure 1. The two regions of OW field pointings along the Galactic plane (dashed line) and the Galactic Bulge region (right). We have colour coded fields to indicate in which Semester the data were obtained.
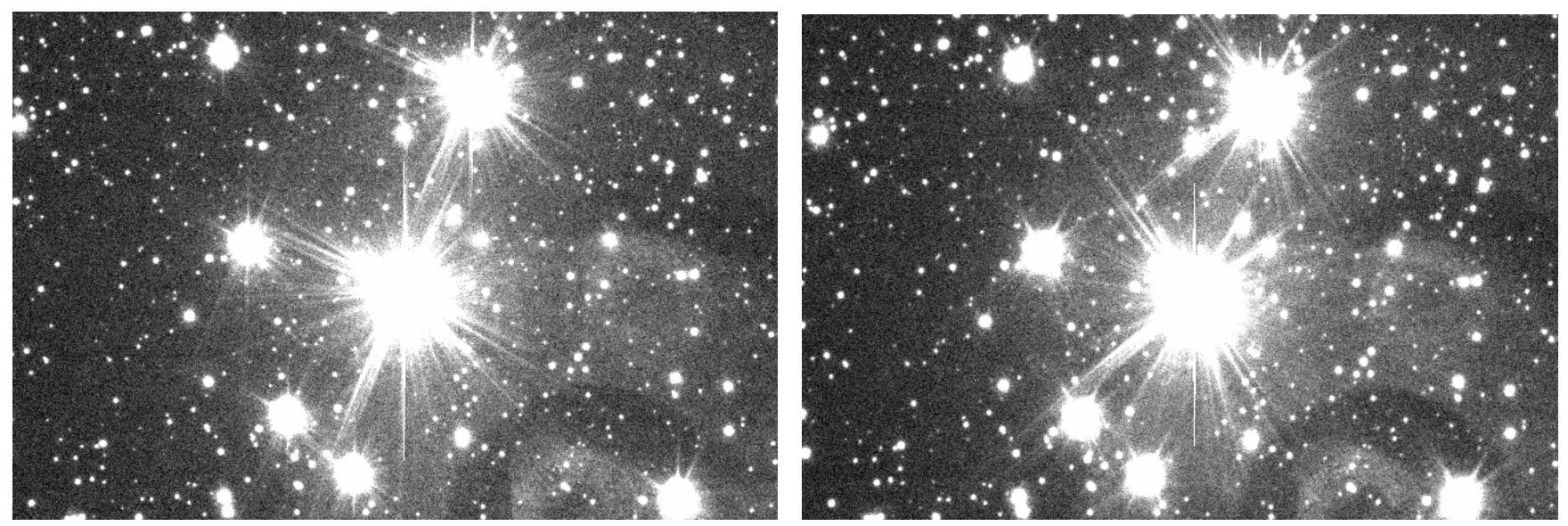

Figure 2. Two images from Field1a, Chip71 taken in Semester 88, (center of the image is $\left.\alpha_{J 2000.0}=07: 40: 26, \delta_{J 2000.0}=-30: 04: 18\right)$ where the rotation of the diffraction spikes relative to the detector is clear. The effect of these spikes sweeping across stars can result in spurious variability.

a set of master flat fields which were made from observations obtained during the month of the science observations. An astrometic solution is obtained for each image using ASTROMETRY.NET software (Lang et al. 2010). The typical residual to the 2MASS positions is $\sim 0.1 \operatorname{arcsec}$ (see Paper I for details).

(ii) Since our fields lie within $\sim 5$ degrees of the Galactic Plane, many (but not all) of the fields are crowded. To account for this and to accommodate variations in seeing, we use the Difference Image Analysis Package DIAPL2 (Wozniak $2000)$ to obtain our photometry. Most wide field photometric surveys show systematic trends in the light curves (i.e. they all show the same long term features) which are due to variations in seeing, transparency and airmass. We detrend our light curves using the SYSREM algorithm (Tamuz, Mazeh \& Zucker 2005). We place the magnitude of each star (OWg) on to the standard (Vega) system by cross-calibrating the reference image for each field with the AAVSO Photometric All-Sky Survey (APASS) $g$ band all-sky catalogue (Henden et al. 2012). (iii) Paper I described a method to flag stars which have been falsely identified as variable stars. The source of these false positives include proximity to the detector edge, bad pixels or saturated stars, or have local high background. The origin of the latter effect can be due to diffraction spikes from saturated stars. Since the VST has an altitude-azimuth mounting, these spikes move over the $2 \mathrm{~h}$ time interval that the observations are made (see Figure 2 for an example). This can inject a spurious modulation into light curves. There is also some movement of stars over the detector (we do not auto-guide the telescope), which can cause stars to cross areas of bad or hot pixels.

(iv) Photometric variability parameters are determined for all light curves using the VARTOOLS package (Hartman et al. 2008). These parameters include: the RMS, $\chi_{\nu}^{2}$, the period $\left(\mathrm{P}_{L S}\right)$ corresponding to the highest peak in the Lomb Scargle (LS) Power spectrum (Lomb 1976, Scargle 1982, Zechmeister \& Kürster 2009, Press et al. 1992), and its associated False Alarm Probability (FAP), and the period and associated FAP from the Analysis of Variance (AoV) period 
search algorithm (Schwarzenberg-Czerny 1989, Devor 2005) which identifies a period by folding the data on a number of trial periods and splits the folded data into different phase bins. The FAP gives a measure of how likely a peak in a periodogram is due to random noise in a light curve. For the LS analysis we searched in frequency space between the Nyquist frequency and the frequency corresponding to the duration of the light curve (these parameters varied by a small degree from field-to-field). Variable objects are then selected on a field by field basis using the distribution of stars in the $\mathrm{P}_{L S}$, FAP plane. We outline how we identify variable stars in the next section.

\section{IDENTIFYING VARIABLE STARS}

How to best identify variable stars among a sample of stars has been explored by many authors (see e.g. Graham et al. 2013). Specific variability tests are best suited to find particular types of variable star. Perhaps the most straightforward method is to select them according to the RMS of their light curve as a function of magnitude - variable objects will have a higher RMS compared to non-variable objects with a similar magnitude. For stars which show periodic flux variations, the LS periodogram is efficient in identifying them. Since we are aiming to find periodic variables we primarily use the LS test.

Our primary method for identifying periodic variable stars relies on the distribution of stars in the $\mathrm{P}_{L S}$, FAP plane. We note that large samples will have a correspondingly large number of false positives purely from statistical reasons. For instance, there are $4.1 \times 10^{6}$ light curves which have $\mathrm{P}_{L S}<20 \mathrm{~min}$. If we were to apply a threshold of $\log _{10}$ (FAP) $<-2.5$ (i.e. a probability of 0.316 percent of being a false positive), we would expect 13110 false positives (out of $4.1 \times 10^{6}$ light curves). Rather than using a fixed threshold of $\log _{10}$ (FAP) to provide an initial selection of candidate variables, we used the Median Absolute Deviation (MAD) to provide a means of identifying stars which are 'outliers' in the $\mathrm{P}_{L S}$, FAP plane (c.f. Figure 3). The MAD statistic is defined for a batch of parameters $\left\{x_{1}, \ldots, x_{m}\right\}$ as

$$
\operatorname{MAD}=\operatorname{median}\left(\left|x_{i}-\operatorname{median}\left(x_{i}\right)\right|\right)
$$

where $x_{i}$, for our purposes, represents values of the FAP (each with a corresponding value of $\mathrm{P}_{L S}$ ). The data are sorted according to $\mathrm{P}_{L S}$ and put into $2 \mathrm{~min}$ period bins and the MAD of the FAP in each bin determined. We use a multiplier, $n$, to select samples which are progressively more distant from the local median FAP of points in each time bin. We do not have an a priori knowledge of which value of $n$ is the most appropriate to use. We therefore identify four samples with $\mathrm{n}=5,10,15$, and 20 which obey $\log _{10}(F A P)<$ Median $\left(\log _{10} \mathrm{FAP}\right)-\left(\mathrm{MAD}\left(\log _{10} \mathrm{FAP}\right) \times n\right)$. This test is done on a field by field basis. For brevity we use the term "MADn sample" to indicate which value of $n$ was used.

As can be seen in Figure 3, which shows the distribution of the MAD5, MAD10, MAD15 and MAD20 samples, larger values of $n$ gives fewer stars - i.e. they are variables with the highest probability. We show the number of stars in different period ranges as a function of different values of $n$ in Table 2. As can also be seen from Figure 3 the distribution of all but the MAD20 sample is highly non-uniform. There are several reasons for this. Firstly, there are intrinsically more variables with long periods compared to short periods (for instance $\delta$ Sct variables which have periods typically greater than half an hour are one of the most populous of all variable stars). Secondly, if systematic trends have been imperfectly removed, longer period trends may remain in the light curves. There are a significant number of stars with $\mathrm{P}_{L S}<20 \mathrm{~min}$ - these are the stars we are most interested in.

Figure 4 shows the distribution of the four samples in the period, OWg plane. Compared to the MAD5 sample, the MAD15 sample has fewer stars which are faint and have long periods. We expect that this is due to many relatively faint objects in the MAD5 sample which have light curves which contain residual systematic trends, or have been affected by diffraction spikes from bright stars. In contrast, the MAD15 sample shows an enhancement of relatively bright stars with periods in the range of 40-70 min: this is likely due to stellar pulsators. At the short period end of the distribution there is a concentration of faint stars which is where we expect $\mathrm{UCBs}$ to be present.

Figure 5 shows the amplitude of the periodic modulation as a function of magnitude for the four samples. We find that for fainter stars the amplitude of intrinsic variability needs to be progressively higher to beat the noise and be selected. The MAD15 sample shows a concentration of low amplitude and relatively bright stars which is likely due to $\delta$ Sct stars.

Figure 6 shows the cumulative distribution of these samples. Using the full period range, a KS test suggests that these distributions do not come from the same parent population. If we apply the same test using an upper limit of $40 \mathrm{~min}$ to the period distributions we find that the MAD5 and MAD10 samples are consistent with coming from the same parent population. On the other hand, if we apply an upper limit of $20 \mathrm{~min}$, we find that all the distributions are consistent with coming from the same parent population. We therefore use the MAD5 sample as derived through the LS test as a starting point for identifying variable stars with periods $<20 \mathrm{~min}$ (since UCBs in this period range are intrinsically rare) and the MAD10 sample for periods $>20 \mathrm{~min}$. In $\S 9$ we use the MAD15 sample to search for interesting sources which have no VPHAS+ colours.

One consequence of fixing the long period limit of the LS periodogram to the duration of light curve is that long period variables can be missed. (This can be eliminated if we set the limit to 20 percent greater than the duration of the light curve and this will be applied in the analysis of future data). Instead the AoV test allows the identification of variables which have a period longer than $2 \mathrm{~h}$ and also eclipsing variables using a phase binning approach (SchwarzenbergCzerny 1989, Devor 2005). We have identified a set of variable candidates based on the AoV test and applying the same 'MAD' process as outlined above. However, in the interests of space we do not outline a full analysis of these AoV samples here, but later we highlight three blue variables which were identified through this test.

In the next section we show that an additional manual verification stage finds a significant percentage of candidate short period variables identified using the LS test that are likely false positive variables. However, in Paper III, we show that followup photometry of stars which have passed both 
Table 2. The number of variable candidates selected using the Lomb Scargle periodogram in each sample defined by four MAD ' $n$ ' levels. The candidates have been grouped in period ranges appropriate for different broad classes of variable star. We indicate the number of stars which have been flagged as having a high background which is a likely indicator that a bright star is nearby and may have caused the light curve to be affected by rotating diffraction spikes (see Figure 2).

\begin{tabular}{rrrrrrrr}
\hline$n$ & $\begin{array}{r}0-20 \\
(\min )\end{array}$ & $\begin{array}{r}20-40 \\
(\min )\end{array}$ & $\begin{array}{r}40-60 \\
(\min )\end{array}$ & $\begin{array}{r}60-110 \\
(\min )\end{array}$ & $\begin{array}{r}>110 \\
(\min )\end{array}$ & $\begin{array}{r}\text { Total } \\
(\text { stars })\end{array}$ & $\begin{array}{r}\text { Stars with } \\
\text { High Background }\end{array}$ \\
\hline 5 & 1673 & 1495 & 5210 & 20462 & 2553 & 31389 & $5129(16.3 \%)$ \\
10 & 1643 & 1355 & 3318 & 7383 & 518 & 14213 & $2658(18.7 \%)$ \\
15 & 348 & 473 & 983 & 1870 & 112 & 3782 & $613(16.2 \%)$ \\
20 & 14 & 137 & 248 & 482 & 32 & 909 & $140(15.4 \%)$ \\
\hline
\end{tabular}
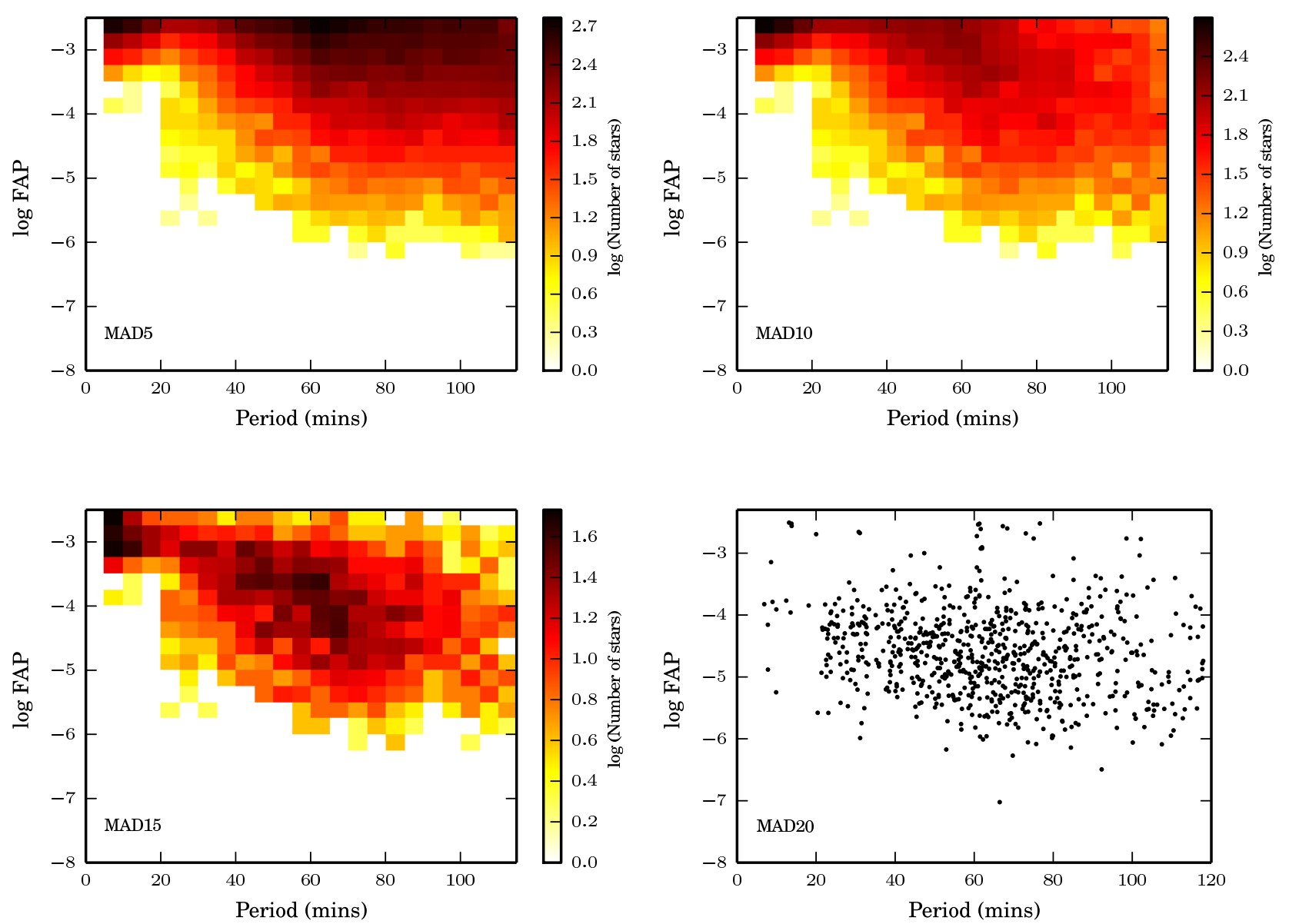

Figure 3. The distribution of variable candidates from Semester 88, 90-94 as function of $\log _{10}$ FAP and period for our four samples (stars which have been automatically flagged as having spurious variability have been removed). The stars are grouped in bins of 5 minute $\times 0.25 \log _{10}$ FAP units. The darker the area, the greater the number of stars it contains. The trends of stars are discussed in the text. Individual stars are shown in the bottom right sample for MAD20. A small number of stars appear with relatively high values of log FAP due to the fact that stars are identified over a 2 min period bin which may be poorly populated.

verification stages confirm the period determined using OW data, thereby verifying that our pipeline can be used to identify short period variable stars, including a reliable estimate of the period.

\section{SEARCHING FOR ULTRA COMPACT BINARIES}

AM CVn binaries together with other compact objects (e.g. single white dwarfs, cataclysmic variables $(\mathrm{CVs})$ ) are located in a distinct region of the $g-r, u-g$ colour-colour plane (see e.g. Carter et al 2013). ${ }^{1}$ Since the OW survey obtains

1 As the VPHAS+ photometry and the OW $g$ mag are on the Vega system, but the SDSS photometry is on the AB system, we 

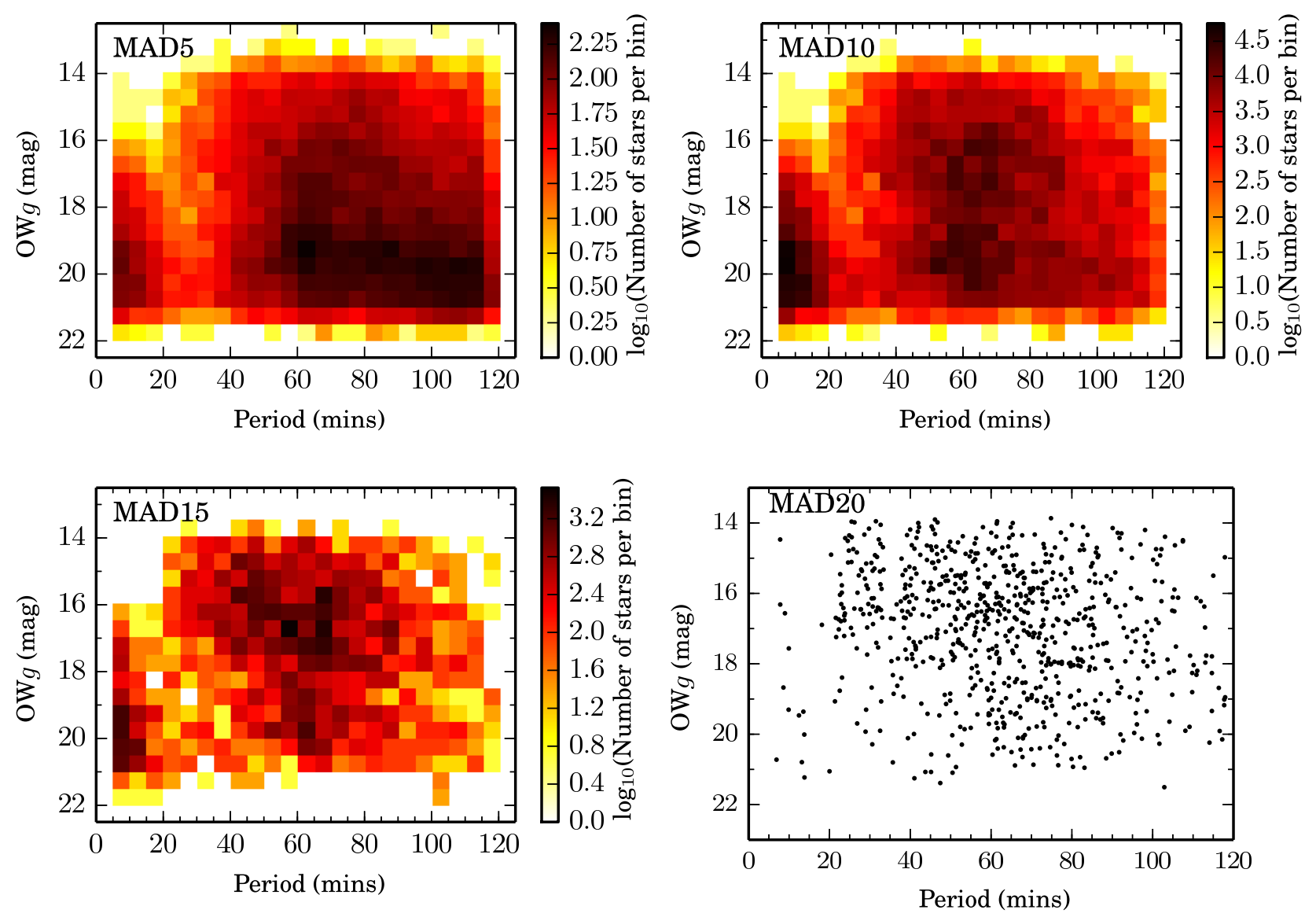

Figure 4. We show the distribution of variable candidates in the Period vs OWg plane for the four samples using the same procedure as in Figure 3 (stars which have been automatically flagged as having spurious variability have been removed). The stars are grouped in bins of 5 minute $\times 0.5$ mag. The darker the area, the larger the number of stars it contains. The trends of faint variable stars are explained in the text.

observations in the $g$ band only, we obtain colour information from the VPHAS+ survey (Drew et al. 2014) whose footprint covers the southern Galactic plane in the same manner as the OW survey.

Given the rarity of AM CVn stars and other UCBs, the MAD5 sample (which gives the greatest number of stars, but also the greatest number of false positives) has been used as the primary sample to search for blue variable objects. Before the filtering or colour selection stages there are 31388 objects and after the automatic flagging procedure there are 26259 objects which remain, with the vast majority being flagged as they are close to a bright star and hence their light curves may have been affected by diffraction spikes. We have used the VPHAS+ second data release (DR2) and made an initial colour selection of $u-g<0.0$ (where we have included stars whose colour meets this condition within the error on its colour index). After this colour cut we have a provisional set of 39 blue short period variable candidates.

Given this is a small number of candidates, we were able to perform a manual verification procedure which con-

transform SDSS colours of specific stars onto the Vega systems using the equations of Blanton \& Roweis (2007). sisted of visually inspecting the light curves, power spectra and their location on the detector. We find that of the 39 blue candidate variables, 22 (56 percent) had to be removed from our list. Most of the stars have been removed because they appear 'swept' by diffraction spikes over the course of the $2 \mathrm{hr}$ series of observations which affects the brightness of the star and the local background (see Figure 2). There were several cases where more than one star was located on the same chip with a similar shape of light curve indicating a systematic trend was still present in the data. This finding indicates that there are still a large number of false positives in the sample selected automatically, which have not been flagged by the pipeline discussed in $\S 3$. Work is on-going to improve the efficiency of the pipeline to flag spurious variables in an automatic manner. We show the details of the blue variable stars which have passed the manual verification stage in Table 3, the location in the $g-r, u-g$ and $r-i, r-H \alpha$ planes in Figure 7, and their light curves in Figure 8. Finding charts for these stars are shown in Figure A.

Of the 17 stars which were identified using the LS test and shown in Table 3, four have $P_{L S}<40$ min, making them prime UCB candidates. 

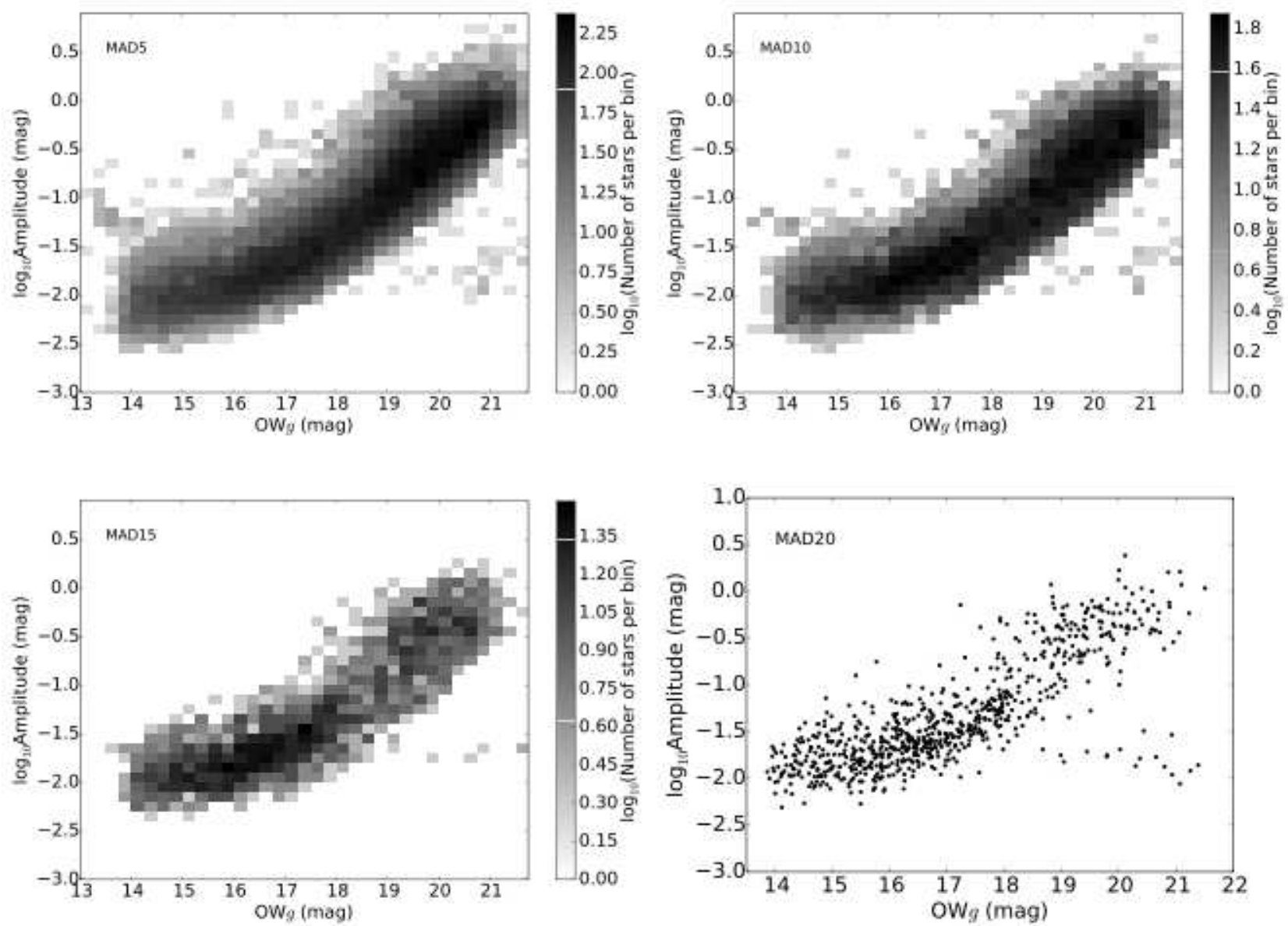

Figure 5. We show the distribution of variable candidates in the $\mathrm{OW} g$ vs Amplitude plane for the four samples using the same procedure as in Figure 3 (stars which have been automatically flagged as having spurious variability have been removed). The stars are grouped in bins of $0.25 \mathrm{mag} \times 0.1 \log _{10}$ Amplitude units. The darker the area, the larger the number of stars it contains. The trends of faint variable stars are explained in the text.

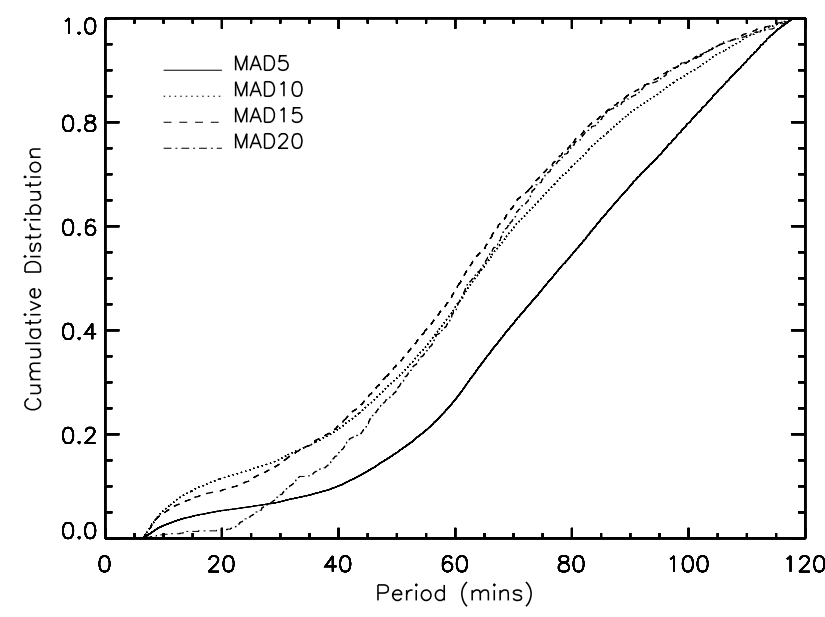

Figure 6. The cumulative distribution of $P_{L S}$ for the samples defined by $\mathrm{n}=5,10,15$ and 20 . Using the full range of period a KS test indicates that they do not come from the same parent population.

- OW J074106.0-294811.0, which shows a period of 22.6 min in the OW light curve, is one of the bluest variable star outlined in Table 3. We presented an optical spectrum of this star obtained using SALT in Paper I, which showed $\mathrm{H} \alpha$ as a very weak absorption line, while $\mathrm{H} \beta$ and $\mathrm{H} \gamma$ showed stronger absorption lines. Further observations of this star have allowed us to identify this object as a UCB with an orbital period of $44 \mathrm{~m}$. It appears to be a very rare UCB in that it is composed of an $\mathrm{sdO} / \mathrm{sdB}$ star plus an unseen companion (Kupfer et al in prep.).

- We do not currently have an optical spectrum for OW J181038.5-251608.6 ( $\left.P_{L S}=28.9 \mathrm{~min}\right)$ although its colours $(g-r=1.11, u-g=-0.50)$ place it at some distance from the unreddened main sequence or white dwarf tracks (Figure 7). There is no known X-ray source within 20 arcsec of its optical position.

- Followup spectra of OW J175358.8-310728.9 $\left(P_{L S}=35.2 \mathrm{~min}\right)$ indicate that this very blue star is a DQ (carbon rich) white dwarf (Macfarlane et al. in prep).

- We show a spectrum of OW J075527.6-314825.2 $\left(P_{L S}=38.6 \mathrm{~min}\right)$ in Paper III from which we infer a spectral type of F6/F7. Its colours $(g-r=0.64, u-g=-0.18)$ are slightly displaced from the main sequence (it has a bluer $u-g$ colour) which may imply another stellar component to the system.

As indicated in $\S 3$ we also identified samples of variable stars using the AoV phase binning test. For the AoV sample which contained the stars whose light curve were the statistically most variable, three stars were bluer than the main sequence and our manual verification processes confirmed the period found from the AoV test. Although their periods lie in the range $85-120 \mathrm{~min}$, and are therefore not UCBs, they show that we can identify short period eclipsing binaries and we outline their details in Table 3 and Figure 7 with their finding charts shown in Figure A. 
In the following sections we discuss these variables together with some of the other blue variable stars identified in Table 3. In addition we examine the observational characteristics of the $\delta$ Sct type stars identified in our survey and some of the previously known variables which are in the OW fields.

\section{HOT SINGLE STARS}

OW J075719.9-292955.5 ( $\left.P_{L S}=98.7 \mathrm{~min}\right)$, has very blue colours $(g-r=0.14, u-g=-0.93)$ but also shows an $r-H \alpha$ index which implies a deep $\mathrm{H} \alpha$ absorption line (Figure 7). Its period suggests that it is unlikely to be a pulsating DA white dwarf but could be a $\mathrm{sdO} / \mathrm{sdB}$ or GW Vir pulsator. It could alternatively be the signature of the rotation period of a white dwarf (we show followup spectra of this star in Paper III).

OW J075305.7-301208.1 ( $\left.P_{L S}=102.2 \mathrm{~min}\right)$, has very blue colours $(g-r=0.14, u-g=-0.93)$ which are close to the unreddend DA white dwarf track shown in Figure 7 . We show in Paper III its optical spectrum which is similar to that of an $\mathrm{O}$ or a B-type star. Further spectra with higher signal-to-noise are planned to determine its nature.

\section{ACCRETING BINARIES}

Three of the stars shown in Table 3 and in Figure 7 have colours indicating $\mathrm{H} \alpha$ in emission. Two of these have a period greater than 90 min suggesting that they are typical hydrogen accreting binaries. A third (OW J174903.9-243720.4) has a period of $55.6 \mathrm{~min}$ which, if this could be verified as the orbital period, would place it well below the CV orbital period minimum, potentially in the group of helium rich CVs (see Breedt et al. 2014 and references therein). It also shows a significant difference in brightness between the epoch of the OW observations and the VPHAS+ epoch ( $\Delta g=3.4 \mathrm{mag}$ ) indicating it is an outbursting system.

OW J180156.3-272256.2 (V5627 Sgr) has strong $\mathrm{H} \alpha$ emission but only moderately blue colours $(g-r=1.17$, $u-g=-0.18$ ). Indeed, it was identified as a suspected nova using data taken by the MACHO project (which aimed to search for dark matter in the form of massive compact hallo objects, i.e. MACHOs; see e.g. Alcock et al 2000) since its brightness declined from $V \sim 15.1$ to 16.9 mag over $\sim 1600$ days (see Mróz et al. 2015 and references therein). Woudt, Warner \& Spark (2005) obtained high speed photometry when the star had a mean $V \sim 16.92$. Although it showed considerably variability, they found a stable period of 2.8 $\mathrm{h}$ which they took to be the orbital period. The OW light curve shown in Figure 8 shows a trend but no evidence of a repeated feature (the duration of the light curve was less than $2.8 \mathrm{hr}$ ). The mean magnitude was $\mathrm{OW} g=18.1$ suggesting the brightness of the star has continued to decline from its outburst.

The bluest of these three accreting objects (OW J080311-291145) has a high amplitude of variability (0.5 mag). The light curve shown in Figure 8 is derived using the difference imaging technique that we use in our pipeline. To investigate this source in more detail we obtained differential aperture photometry using the OW data. The light curve of this source is shown in the top panel of Figure 9 and a clear short duration $(<10.7 \mathrm{~min}$ ) eclipse is seen. (The cadence of our observations preclude a more precise estimate of the eclipse duration). The reason that the photometric points during eclipse were not recorded in the differential imaging light curve is due to the fact that we place a $3 \sigma$ lower limit for detecting stars in an image. (Since this may have implications for identifying other eclipsing systems, we are re-assessing this part of the pipeline). Paper III shows its optical spectrum which confirms this as a high inclination CV.

The OW data implies that the orbital period of OW J0803 is greater than $\sim 60 \mathrm{~min}$. For comparison the magnetic eclipsing CV UZ For has an orbital period of 126.6 min and an eclipse duration of $\sim 0.063$ cycles (e.g. Bailey \& Cropper 1991). Taking an upper limit of $10.7 \mathrm{~min}$ as the duration of the eclipse in OW J080311-291145, this suggests by comparison with UZ For, an orbital period of $\sim 170 \mathrm{~min}$. If the eclipse duration was as short as $9 \mathrm{~min}$, this would imply an orbital period of $\sim 140 \mathrm{~min}$. Although there is a great deal of uncertainty on the orbital period of this new CV, it is potentially interesting in that it may reside in the $2-3 \mathrm{~h}$ CV orbital period gap (see Zorotovic et al. 2016 for a recent discussion of the period gap). Photometric observations are strongly encouraged to determine the orbital period of this eclipsing CV.

\section{A POSSIBLE NON-INTERACTING, SHORT PERIOD, ECLIPSING SYSTEM}

In the previous section we showed that OW J080311-291145 has been identified as a CV through its VPHAS+ colours and the eclipse in its OW light curve. We identified OW J181013.7-213828.3 as a variable through the AoV test and find that it shows two eclipse-like features with one possible secondary eclipse (Figure 8). If this result can be confirmed, then it would be a binary with an orbital period of $\sim 85 \mathrm{~min}$. The VPHAS+ colours do not indicate $\mathrm{H} \alpha$ in emission and this is confirmed from our spectra shown in Paper III. Although the mean magnitude of OW J181013.7 during the OW observations differs by $\sim 1.1$ mag compared to the VPHAS $+g$ band magnitude, this is greater than the depth of the eclipse like feature seen in the OW photometry. Further observations are required to confirm the eclipse-like features and search for changes in its mean long term brightness which would be an indicator of sporadic mass transfer.

\section{$8 \delta$ Sct-TYPE PULSATORS}

The seven stars shown in Table 3 and Figure 7 have colours consistent with that of stars whose intrinsic colours are close to that of main sequence stars of A/F spectral type, or stars with low reddening, or with intrinsically blue stars which have been heavily reddended. They all show very low amplitude modulation ( $<0.02 \mathrm{mag}$ ). We currently have optical spectra for two of these stars and they indicate a spectral type F6-F8 (Paper III). The most likely scenario is that these are $\delta$ Sct type stellar pulsators.

Indeed, surveys with a similar strategy to OW have been successful in identifying short period stellar pulsators 

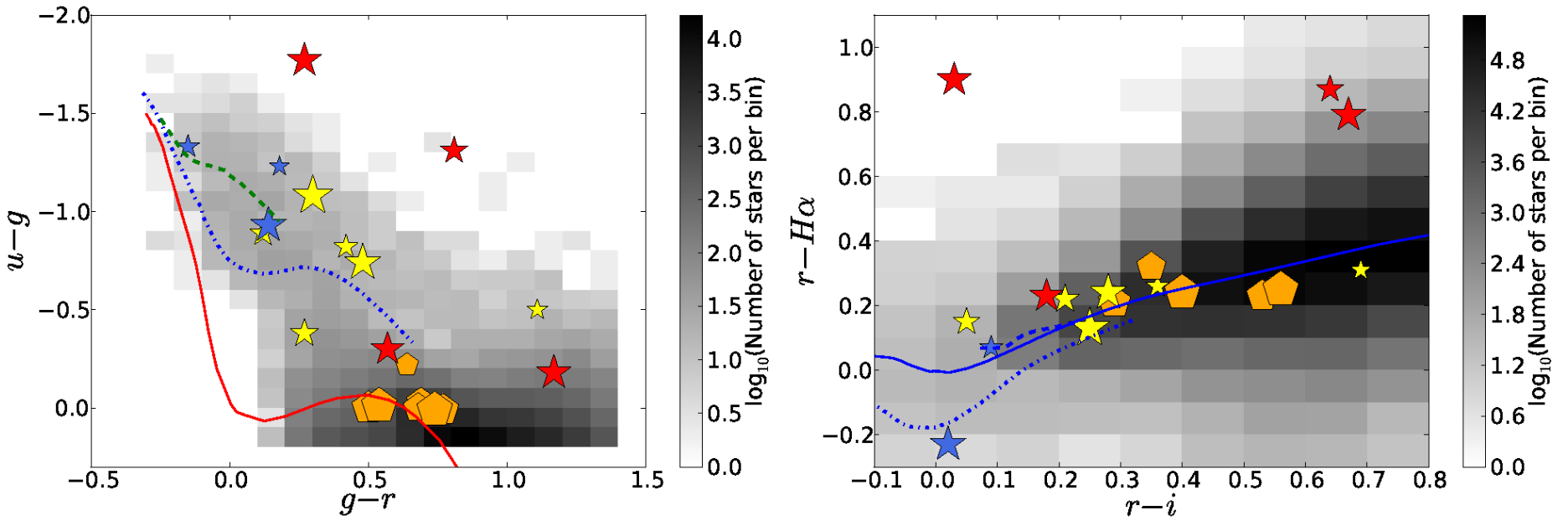

Figure 7. The $g-r, u-g$ (left) and $r-i, r-H \alpha$ (right) colours of the variable stars shown in Table 3 . The red line marks the unreddened main sequence (taken from Drew et al. 2014), the blue line marks the cooling track for DA white dwarfs with $\log g=8$ and the green line the cooling track for DB white dwarfs with $\log g=8$ (both taken from Raddi et al. 2016). The size of the symbol increases as a function of period of the variable. Red refers to CVs, accreting objects or eclipsing binaries; blue represents UCB, DQ or DA-like white dwarf; $\delta$ Sct type stars are shown as orange polygons and currently unknown objects are yellow stars. The pixelated grayscale map shows the number of stars (whether variable or not) in the OW survey with VPHAS+ colours.
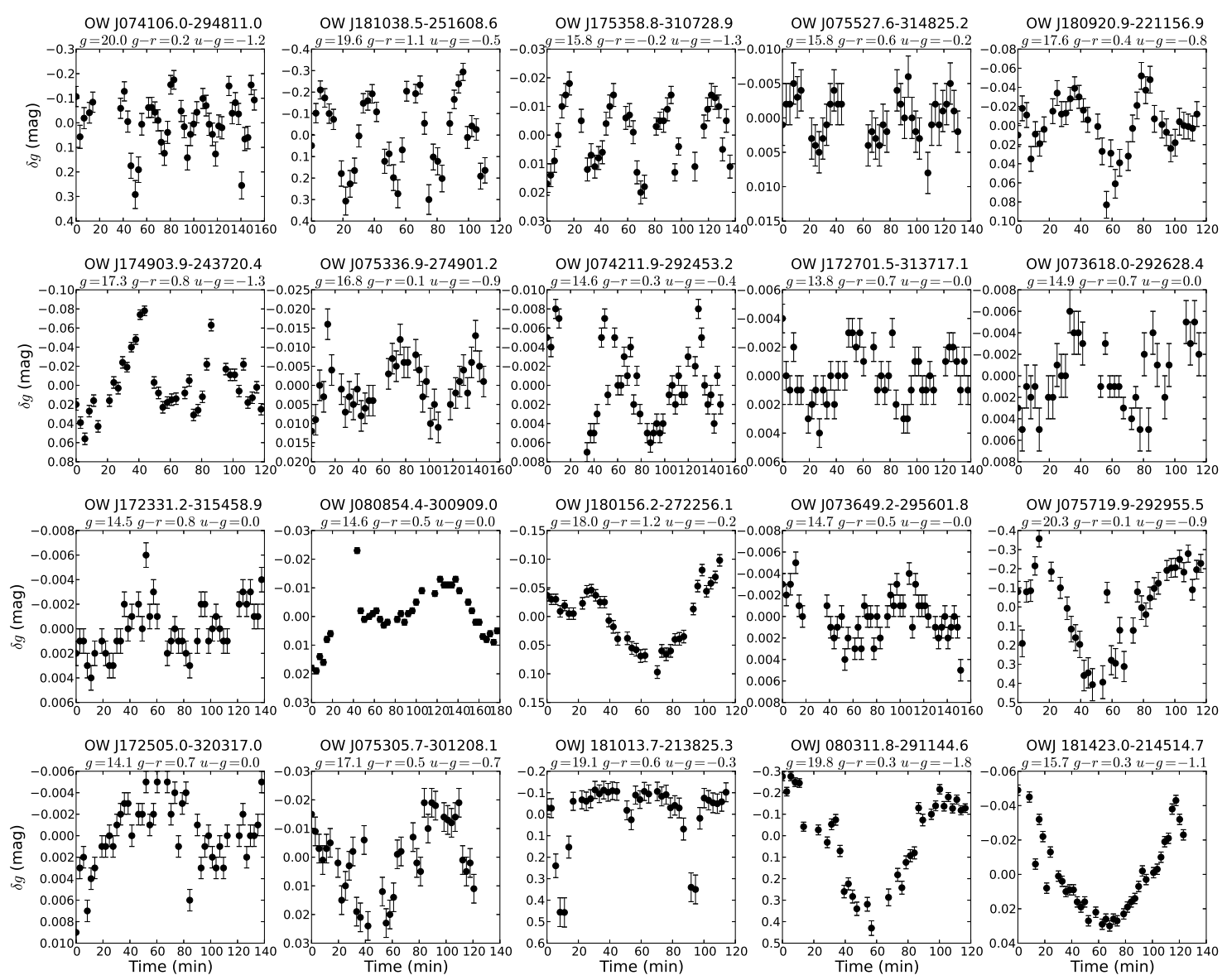

Figure 8. Light curves of the 20 blue variable stars shown in Table 3 and whose colours are shown in Figure 7 . We show their name, OW $g$ mag, $g-r$ and $u-g$ colour indices above each light curve. 
Table 3. The 20 blue stars which were identified as variable in the MAD5 sample and subsequently passed the manual verification stage. We give the sky co-ordinates, the period corresponding to the most prominent peak in the LS power spectrum and its corresponding False Alarm Probability (FAP), the OW $g$ mag and amplitude of the variation, the colours derived from VPHAS+ data and the difference between the $g$ mag derived in the OW $g$ data and the VPHAS + data $(\mathrm{OWg}-\mathrm{Vg})$. The lower three stars were identified by means of the AoV test and give the period and FAP of the most prominent period. Notes: (1) PaperI; (2) Kupfer et al. (in prep); (3) Macfarlane et al. (in prep); (4) Paper III.

\begin{tabular}{|c|c|c|c|c|c|c|c|c|c|c|c|c|}
\hline Name & $\begin{array}{l}\text { RA } \\
\text { (J2000) }\end{array}$ & $\begin{array}{l}\text { Dec } \\
(\mathrm{J} 2000)\end{array}$ & $\begin{array}{r}P_{L S} \\
(\mathrm{~min})\end{array}$ & $\begin{array}{r}\text { LS } \\
\text { FAP }\end{array}$ & $\begin{array}{l}\mathrm{OWg} \\
(\mathrm{mag})\end{array}$ & $\begin{array}{r}\text { Amp } \\
(\mathrm{mag})\end{array}$ & $u-g$ & $g-r$ & $r-i$ & $r-H \alpha$ & $\begin{array}{r}\text { OWg- } \\
\text { VPHASg }\end{array}$ & Type \\
\hline OW J074106.0-294811.0 & $07: 41: 06.0$ & $-29: 48: 11.0$ & 22.6 & -3.94 & 20.03 & 0.224 & -1.23 & 0.18 & 0.05 & & -0.03 & $\mathrm{UCB}(1,2)$ \\
\hline OW J181038.5-251608.6 & $18: 10: 38.5$ & $-25: 16: 08.6$ & 28.9 & -4.88 & 19.62 & 0.423 & -0.50 & 1.11 & 0.69 & 0.31 & -0.27 & \\
\hline OW J175358.8-310728.9 & $17: 53: 58.8$ & $-31: 07: 28.9$ & 35.2 & -4.72 & 15.81 & 0.026 & -1.33 & -0.15 & 0.09 & 0.07 & 0.08 & DQ White dwarf(3) \\
\hline OW J075527.6-314825.2 & $07: 55: 27.6$ & $-31: 48: 25.2$ & 38.6 & -3.17 & 15.84 & 0.007 & -0.18 & 0.64 & 0.40 & & -0.10 & $\delta$ Sct like $(4)$ \\
\hline OW J180920.9-221156.9 & $18: 09: 20.9$ & $-22: 11: 56.9$ & 44.7 & -2.57 & 17.57 & 0.056 & -0.82 & 0.42 & 0.36 & 0.26 & 0.68 & \\
\hline OW J174903.9-243720.4 & $17: 49: 03.9$ & $-24: 37: 20.4$ & 55.6 & -3.28 & 17.31 & 0.071 & -1.31 & 0.81 & 0.64 & 0.87 & -3.44 & $\mathrm{CV}$ \\
\hline OW J075336.9-274901.2 & $07: 53: 36.9$ & $-27: 49: 01.2$ & 59.4 & -2.60 & 16.76 & 0.014 & -0.89 & 0.12 & 0.05 & 0.15 & 0.04 & \\
\hline OW J074211.9-292453.2 & $07: 42: 11.9$ & $-29: 24: 53.2$ & 61.1 & -3.14 & 14.62 & 0.008 & -0.38 & 0.27 & 0.21 & 0.22 & 0.16 & \\
\hline OW J172701.5-313717.1 & $17: 27: 01.5$ & $-31: 37: 17.1$ & 63.2 & -2.57 & 13.83 & 0.003 & -0.03 & 0.69 & & 0.22 & 0.03 & $\delta$ Sct like \\
\hline OW J073618.0-292628.4 & $07: 36: 18.0$ & $-29: 26: 28.4$ & 65.9 & -2.58 & 14.85 & 0.006 & 0.00 & 0.68 & 0.35 & 0.32 & -0.06 & $\delta$ Sct like \\
\hline OW J172331.2-315458.9 & $17: 23: 31.2$ & $-31: 54: 58.9$ & 75.3 & -2.75 & 14.54 & 0.004 & 0.01 & 0.77 & 0.53 & 0.23 & -0.09 & $\delta$ Sct like \\
\hline OW J080854.4-300909.0 & 08:08:54.4 & $-30: 09: 09.0$ & 84.4 & -2.66 & 14.63 & 0.018 & 0.00 & 0.50 & 0.29 & 0.21 & -0.10 & $\delta$ Sct like \\
\hline OW J180156.2-272256.1 & $18: 01: 56.2$ & $-27: 22: 56.1$ & 92.4 & -4.11 & 18.05 & 0.128 & -0.18 & 1.17 & 0.67 & 0.79 & -0.07 & CV, V5627 Sgr \\
\hline OW J073649.2-295601.8 & $07: 36: 49.2$ & $-29: 56: 01.8$ & 95.8 & -3.66 & 14.66 & 0.004 & -0.01 & 0.54 & 0.40 & 0.24 & 0.00 & $\delta$ Sct like \\
\hline OW J075719.9-292955.5 & $07: 57: 19.9$ & $-29: 29: 55.5$ & 98.7 & -3.46 & 20.27 & 0.440 & -0.93 & 0.14 & 0.02 & -0.23 & -0.11 & $\mathrm{DA} W \mathrm{WD} / \mathrm{SdB} ?(4)$ \\
\hline OW J172505.0-320317.0 & $17: 25: 05.0$ & $-32: 03: 17.0$ & 99.9 & -2.88 & 14.06 & 0.006 & 0.01 & 0.74 & 0.56 & 0.25 & -0.07 & $\delta$ Sct like \\
\hline OW J075305.7-301208.1 & $07: 53: 05.7$ & $-30: 12: 08.1$ & 102.2 & -3.28 & 17.06 & 0.030 & -0.74 & 0.48 & 0.28 & 0.24 & 0.00 & O type spectrum (4) \\
\hline Name & $\begin{array}{l}\text { RA } \\
(\mathrm{J} 2000)\end{array}$ & $\begin{array}{l}\text { Dec } \\
(\mathrm{J} 2000)\end{array}$ & $\begin{array}{l}P_{A o V} \\
(\mathrm{~min})\end{array}$ & $\begin{array}{l}\text { AoV } \\
\text { FAP }\end{array}$ & $\begin{array}{l}\mathrm{OWg} \\
(\mathrm{mag})\end{array}$ & $\begin{array}{r}\text { Amp } \\
(\mathrm{mag})\end{array}$ & $u-g$ & $g-r$ & $r-i$ & $r-H \alpha$ & $\begin{array}{r}\text { OWg- } \\
\text { Vg }\end{array}$ & Type \\
\hline OWJ 181013.7-213825.3 & 18:10:13.7 & $-21: 38: 25.3$ & 85.4 & 10.59 & 19.10 & 0.175 & -0.30 & 0.57 & 0.18 & 0.23 & 1.09 & Possible Eclipsing Binary \\
\hline OWJ 080311.8-291144.6 & 08:03:11.8 & $-29: 11: 44.6$ & 90.2 & 15.90 & 19.75 & 0.500 & -1.77 & 0.27 & 0.03 & 0.90 & -0.02 & Eclipsing CV (3) \\
\hline OWJ 181423.0-214514.7 & $18: 14: 23.0$ & $-21: 45: 14.7$ & 118.9 & 17.06 & 15.74 & 0.058 & -1.08 & 0.30 & 0.25 & 0.13 & -0.01 & \\
\hline
\end{tabular}
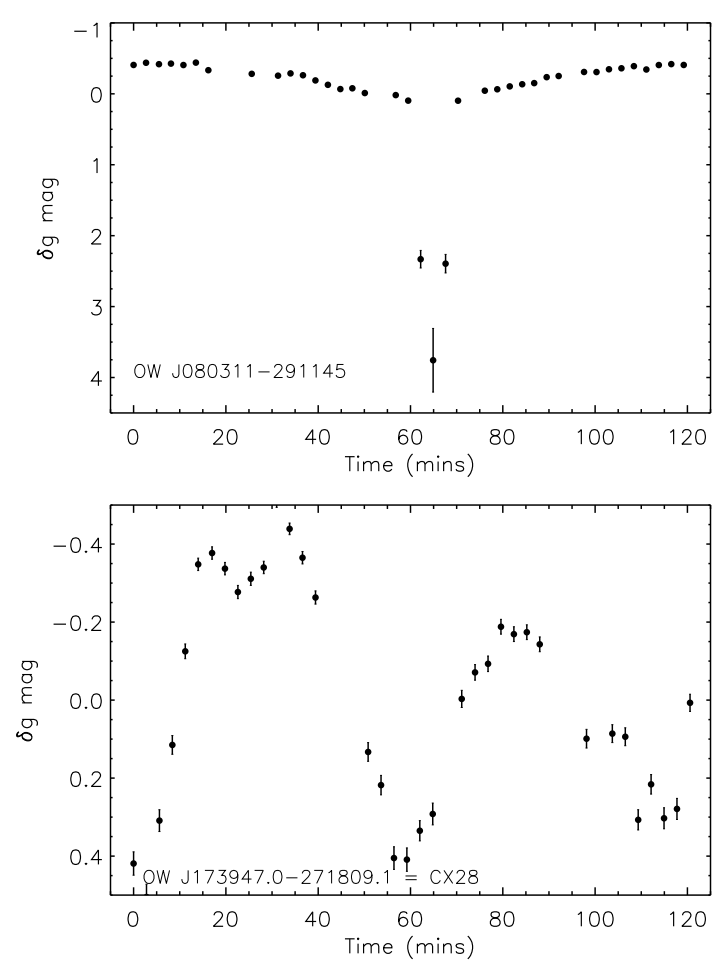

Figure 9. CVs identified in the OW survey. Top panel: The eclipsing CV OW J080311-291145 which was discovered from its OW light curve and followup spectroscopy (Paper III); Bottom panel: OW J173947.0-271809.1 which was identified as a possible Intermediate Polar in the Chandra Bulge X-ray survey (Britt et al. 2013). (e.g. Ramsay et al. 2006, Ramsay et al. 2014). Many of these are $\delta$ Sct type stars with late-A to mid-F spectral type and dominant periods in the range of $\sim 26$ min to $\sim 6 \mathrm{~h}$ (see e.g. Breger 2000 and Chang et al. 2013). Other types of pulsators with similar spectral type include $\gamma$ Dor, roAp and SX Phe stars and only medium resolution spectra with high signal-to-noise can distinguish the specific class of variable star.

To identify a larger sample of candidate $\delta$ Sct type pulsators from the OW survey we took the MAD10 sample as an initial starting point and searched for stars with a period less than $1 \mathrm{hr}$, approximately half the duration of the OW light curve. Given that the fields are close to the Galactic plane, we expect many stars will have colours which are significantly reddended (in contrast to the seven stars which are likely $\delta$ Sct type stars shown in Table 3). In selecting a sample of $\delta$ Sct candidates, we assume they have intrinsic colours in the range A0 and F5. We therefore select all stars which have a $u-g$ colour redder than the main sequence and $u-g, g-r$ colours which are consistent with intrinsic colours of A0-F5 stars. This is best seen in Figure 10 which shows our sample of $\delta$ Sct type stars in the $g-r, u-g$ plane.

There are 5263 variables in the MAD10 sample which have a period of $P_{L S}<60 \mathrm{~min}$ and passed our initial flagging stages. Of these 455 have VPHAS+ colours which are consistent with a star with intrinsic colours of an A0 to F5 star. The significantly smaller number of candidates with both colour indices within the expected range is consistent with the relatively low values of the percentages of stars with colour information (for instance only 23 percent of the 5263 stars currently have a $u-g$ index) due to the incompleteness of the VPHAS+ dataset which is still being obtained. After a manual verification phase we removed 78 stars out of 455 


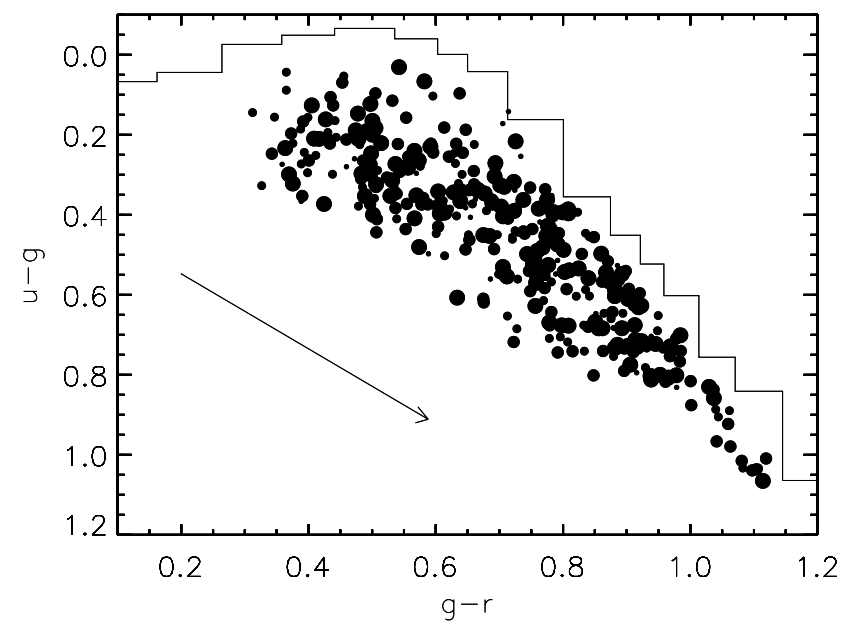

Figure 10. The $g-r, u-g$ colours of the $\delta$ Sct type variables where the size of the symbol increases as a function of period. The arrow represents the direction and magnitude of the reddening vector for $A_{V}=1.0$ and the histogram represents the unreddened main sequence (from Drew et al. 2014).

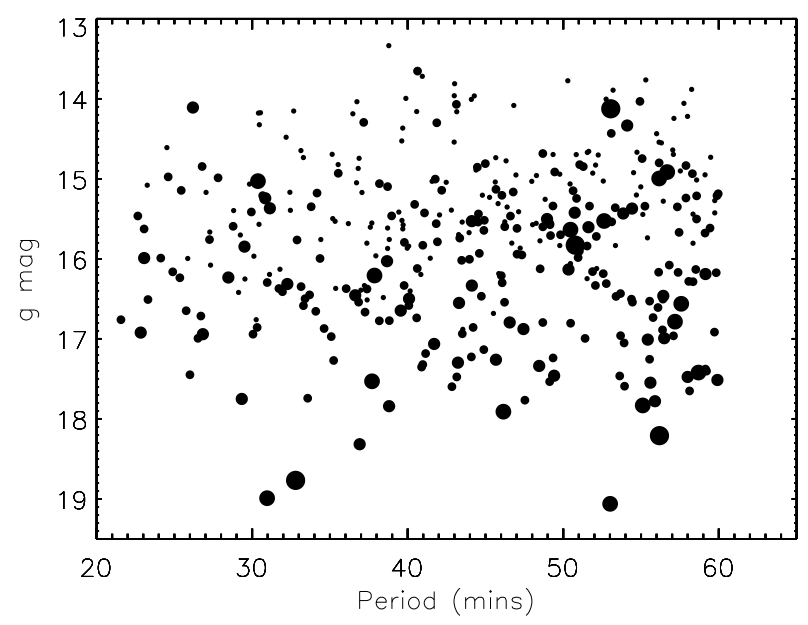

Figure 11. The $\delta$ Sct type pulsators which have been selected due to their colour and variability as a function of period and $g$ mag. The size of the symbol reflects the amplitude of the variability $(<0.01 \mathrm{mag}$ for the smallest symbols, with $>0.05 \mathrm{mag}$ for the largest symbols).

stars (18 percent) because their light curves were affected by very close stars or are in the diffraction spikes of a nearby bright star. We show the period and $\mathrm{OWg}$ mag of the remaining stars in Figure 11 where the symbol size reflects the amplitude of variability. Of the 377 stars, 39.8 percent stars have an amplitude less than $0.01 \mathrm{mag}, 1.1$ percent have an amplitude greater than $0.05 \mathrm{mag}$, the highest amplitude being 0.11 mag. The star with the shortest period has a period of $9.3 \mathrm{~min}$. We discuss the characteristics of this sample in $\S 11.3$.
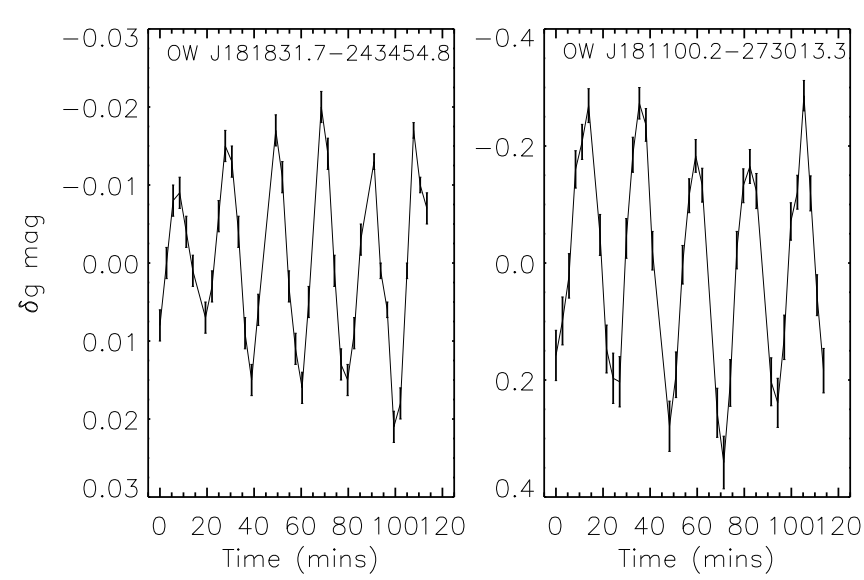

Figure 12. Two examples of short period pulsating candidates which currently have no colour information, left panel OW J181831.7-243454.8 (20.4 min) and right panel OW J181100.2$273013.3(23.1 \mathrm{~min})$.

\section{STARS WITH NO VPHAS+ COLOUR INFORMATION}

Since the VPHAS+ survey is on-going, many of our variable stars do not currently have colour information. For instance, for the variables in the MAD15 sample only 24.0 percent currently have $u-g$ and $g-r$ colours. A set of light curves of short period stars which have no colour information were selected from the MAD15 sample in order to detect potentially new interesting stars which are statistically very likely to be intrinsically variable. The MAD15 sample contains 3168 stars after automatic flagging (c.f. Table 2). Out of these, 2089 stars have no VPHAS $+g$ band information and of these 227 have $P_{L S}<20$ minutes and 260 have $20<P_{L S}<40$ minutes.

We show two examples of short period variables in Figure 12. OW J181831.7-243454.8 (OWg = 14.90) has a period of $20.4 \mathrm{~min}$ and an amplitude of $0.029 \mathrm{mag}$, whilst OW $\mathrm{J} 181100.2-273013.3(\mathrm{OWg}=18.40)$ has a period of $23.1 \mathrm{~min}$ and an amplitude of $0.46 \mathrm{mag}$. The brighter of these two stars has an amplitude consistent with $\delta$ Sct type pulsators at the brighter end of the distribution shown in Figure 11. The stars have a very similar period but the fainter of the two has a much higher amplitude of modulation. Indeed, it is much greater than the $\delta$ Sct type pulsators shown in Figure 10 making it an interesting object for further investigation.

Over the course of the VPHAS+ project (Drew et al. 2014) further sky coverage will be obtained and OW fields which do not currently have colour information will be matched with these additional new VPHAS+ fields. In the mean time, by comparing the period, $g$ mag brightness and amplitude of variable stars with no colour information to those which do have colour information, we will be able to optimally target followup photometry and spectroscopy for stars which have unusual characteristics. 


\section{PREVIOUSLY KNOWN OBJECTS IN OW FIELDS}

To determine which $\mathrm{OW}$ variable objects have information in the literature we took our MAD5 sample after automatic filtering and searched for objects in the SIMBAD database using a 2 arcsec radius (although the mean uncertainty in our positions is typically 0.1 arcsec, applying a larger search radius allows for a small amount of proper motion and uncertainty in the position recorded in the SIMBAD database). We found a total of 179 stars out of the 26260 in the SIMBAD data base. The greatest proportion of stars are RR Lyr stars $(76 / 179)$. Given that the photometric period of these stars is much longer (typically $0.2-1 \mathrm{~d}$ ) then the duration of the OW light curves, it is likely that we are catching only a small fraction of the pulsation period. We also find two CVs, two symbiotic binaries and two planetary nebulae which we now discuss in more detail.

\subsection{Cataclysmic Variables}

There are two known CVs in our MAD5 sample: OW J173947.0-271809.1 and OW J180156.3-272256.2 (V5627 Sgr). We discussed V5627 Sgr in $\S 6$, while OW J173947.0271809.1 was identified as an X-ray source (CX28) in the Chandra Bulge X-ray survey (Jonker et al. 2011). Its Xray properties, coupled with its optical spectrum (Britt et al. 2013), hints at an Intermediate Polar nature (CVs whose accretion disk is truncated and have a white dwarf magnetic field strength $B \sim 10^{5-7} \mathrm{G}$ ). The optical photometry shown in Britt et al. indicates that CX28 is optically variable, with a mean brightness of $g \sim 16.7$, a high $(\sim 0.7 \mathrm{mag})$ amplitude of variations and some (but not conclusive) evidence of a $2.76 \mathrm{hr}$ period. We show the OW photometry of CX28 in Figure 9 where there is some suggestion of a period of $\sim 1$ $\mathrm{hr}$. The star is also significantly fainter at the time of the OW observations (OWg 19.0).

\subsection{Central Stars of Planetary Nebulae}

Searches for variability in the flux of the central stars of Planetary Nebulae (CSPN) have been on-going for decades (e.g. Bond 1979, Miszalski et al. 2009). It is of interest because the observed distribution of orbital periods can be compared to theoretical models of the formation of $\mathrm{PN}$ which predict their period distribution, in particular bipolar/asymmetric PN which are predicted to be formed (perhaps largely) due to binary interactions (e.g. Bond 2000).

Our MAD5 sample includes two stars classified as (possible) Planetary Nebulae. OW J1722353-3214036 (= Th 38 ) shows a modulation on a period of $22.6 \mathrm{~min}$. However, this period could not be verified using differential aperture photometry, perhaps because there is a star in the wings of its PSF. PN Th $2-8(\mathrm{OWg}=15.7)$ is classed in SIMBAD as a planetary nebula, but Acker et al. (1987) finds no emission lines in its optical spectrum casting considerable doubt on this claim (a finding confirmed by our spectrum which is shown in Paper III).

The other PN-like star is OW J1747339-2147231 (= H 2-22) which shows a partial sinusoidal modulation (OWg $=19.2$, amplitude $\sim 0.2 \mathrm{mag}$ ) over its $2 \mathrm{~h}$ light curve. The CSPN with the shortest period appears to be $3.4 \mathrm{~h}$
(Miszalski et al. 2009). If the optical modulation of PN H 2-22 can be confirmed this may place it at the short period end of the CSPN distribution. Further optical photometry of this object is clearly desirable.

\subsection{Symbiotic Binaries}

Symbiotic stars are interacting binary systems containing a red giant star and a hotter component, which is typically a white dwarf (see Mikolajewska 2007 for a review). Recently evidence has accumulated to suggest that symbiotic stars could be progenitors of a fraction of supernovae Ia explosions (e.g. Dilday et al. 2012). Two of our variable candidates are classed as symbiotic binaries in the SIMBAD database. The symbiotic binary SS73 122 (OW J1804412-2709124) shows a periodic modulation on a period of $22.8 \mathrm{~min}$ and an amplitude of $0.006 \mathrm{mag}(\mathrm{OW} g=15.1)$. We obtained differential aperture photometry of this star but were not able to verify the 22.8 min modulation, perhaps because there is a $g=18.6$ mag star $\sim 6$ arcsec distant. Further photometry of this star is required.

The second star, Hen 2-357 (OW J1810439-2757500), shows a modulation on a period of $31.4 \mathrm{~min}$ and an amplitude of $0.008 \mathrm{mag}(\mathrm{OW} g=14.7)$. This period is confirmed using differential aperture photometry and we show its light curve in Figure 13. This would make it only the second known symbiotic binary to show a periodic modulation on a period less than one hour. The most obvious origin of the modulation is a hot accretion region on the white dwarf coming into and out of view as the white dwarf rotates on a period of $31 \mathrm{~min}$ (a time-scale similar to the rotation period of the white dwarfs in CVs). An alternative scenario is that the $31 \mathrm{~min}$ is a quasi-periodic oscillation due to flickering originating from the accretion disk. A much longer series of photometry is required to determine how stable the period is. The optical spectra of both these stars is presented in Paper III and are consistent with spectra of known symbiotic binaries, although there is some evidence for extended emission.

The first symbiotic star to be identified as magnetic was Z And (Sokoloski \& Bildsten 1999) which has an optical light curve which shows a modulation on a period of 28 min. Since then only one other symbiotic system has shown a periodic modulation (BF Cyg at $1.8 \mathrm{~h}$, Formiggini \& Leibowitz 2009). It is not clear if the lack of symbiotic binaries showing evidence for a magnetic field is due to the coherent signal of the rotating white dwarf being greatly diluted by the light from the mass donating giant star, or that it is an indication of their formation (c.f. Yungelson et al. 1995).

\section{DISCUSSION}

We have identified samples of variable stars, some of which contain blue compact stars, and others are likely $\delta$ Sct type pulsating stars. To determine the nature of specific variable stars, we require followup photometry and spectroscopy of individual objects, as we have done in Paper III. 


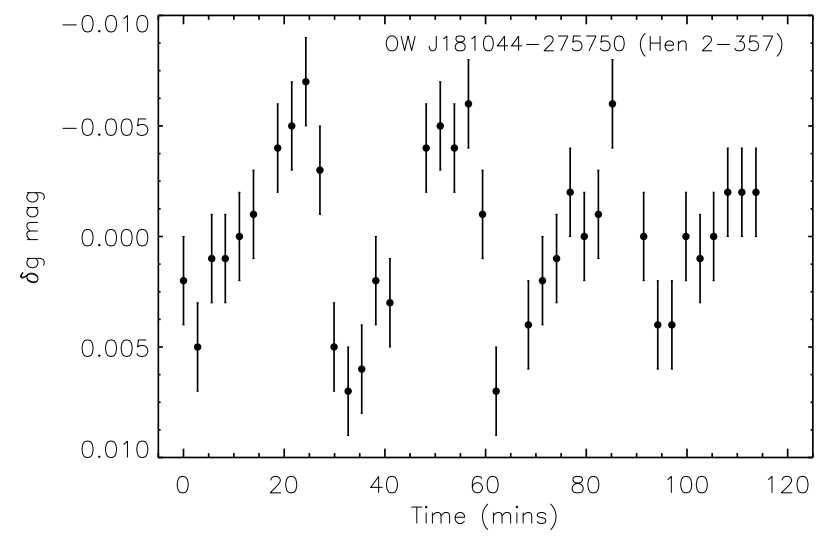

Figure 13. The OW light curve of the Symbiotic Binary Hen 2357 which shows evidence of a periodic modulation on a period of $31.4 \mathrm{~min}$. This may imply that it has a significant magnetic field which would make it only the second known magnetic Symbiotic Binary.

\subsection{The distribution of variable stars}

In Figure 14 we show the distribution of different classes of variable stars in the Period vs $\mathrm{OW} g$ magnitude, $\mathrm{OW} g$ vs Amplitude, and the Period vs Amplitude planes. We find that the blue variables shown in Table 3 which are not $\delta$ Sct types tend to be fainter than $\mathrm{OWg} \sim 16 \mathrm{mag}$ and have $P_{L S}>$ $20 \mathrm{~min}$. The $\delta$ Sct types typically have $P_{L S}>30 \mathrm{~min}$ and are brighter than $\mathrm{OWg} \sim 17$. On the other hand, the stars which are either CVs or eclipsing show amplitudes which are greater than the mean. More compact blue stars show amplitudes which are more typical of other variables making the $\mathrm{OW} g$ vs Amplitude plane less suitable for identifying them. Many of the stars which show short periods and do not have VPHAS+ colour information show a wide range of amplitude. However, the fact that the short period blue objects also show high amplitudes indicates that this is a population well worth exploring for new interesting objects.

\subsection{The number of compact stars}

In its first four years, OW has covered 134 square degrees of sky close to the Galactic plane. Only 45.4 square degrees currently have $u$ and $g$ band information and only 18.4 percent of the MAD5 sample of variables have $u-g$ and $g-r$ colour indices. The number of stars in the $g-r, u-g$ colour-colour plane is illustrated in Figure 7 together with the cooling track for DA and DB white dwarfs with $\log g=8$. Around 900 stars in the OW survey are currently located close to these cooling tracks or along the reddening vectors implying 20 stars per square degree. This is approximately twice the number found in the first 211 square degrees of the UVEX survey where 2170 'UV excess' stars were identified (Verbeek et al. 2012).

A detailed search for DA white dwarfs found between 153 and 211 objects in 211 square degrees of the UVEX survey depending on how the samples were identified (Verbeek et al. 2013). The fact that DA white dwarfs make up a relatively small percentage of stars in the same part of the colour-colour plane is not a surprise but does indicate that
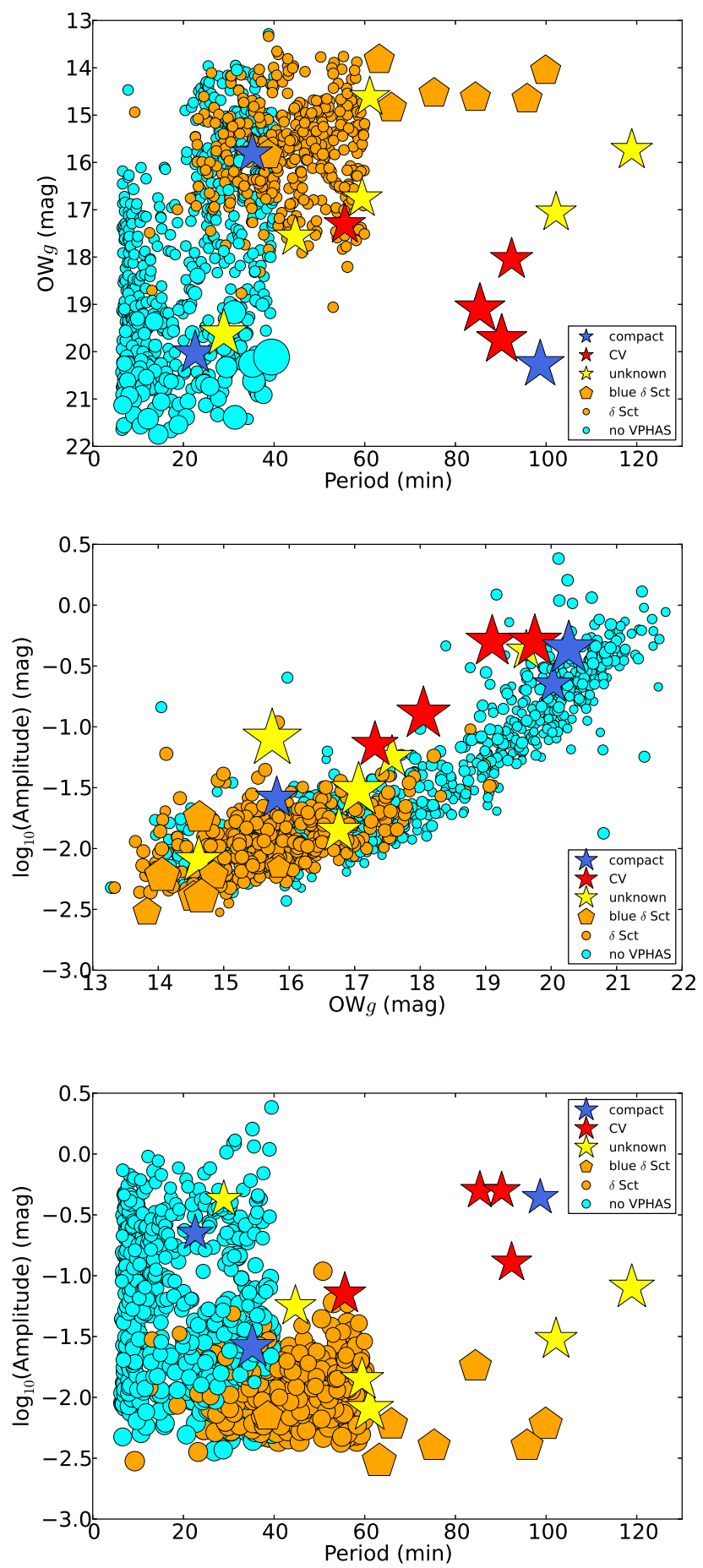

Figure 14. The distribution of different classes of variable stars in the $P_{L S}$, OWg plane (top panel), the OWg, Amplitude plane (middle panel) and $P_{L S}$, Amplitude plane (bottom panel). Variable stars shown in Table 3 and Figure 7 are indicated by the following symbols: blue stars refer to systems with a white dwarf; red stars indicate CVs, accreting objects or eclipsing binaries; yellow stars are variables with currently unknown type, and orange polygons refer to $\delta$ Sct stars. Orange circles refer to $\delta$ Sct stars outlined in $\S 8$ and turquoise circles refer to stars which currently have no VPHAS+ information ( $\$ 9)$. In the upper panel the size of the symbol increases as a function of amplitude, in the middle it increases as a function of period and in the lower panel it increases as the star gets fainter. 
spectroscopic followup is essential in determining the nature of any blue variable star. Our search for variable stars identified around ten stars which are close to the DA and DB cooling track, giving 0.2 variables per square degree.

Differences between the numbers of compact blue objects derived from the UVEX and OW surveys could be due to the fact that the initial release of UVEX data avoided the Galactic Bulge regions, where as around half of the OW fields have been directed towards the Bulge. These issues will be explored in future work.

\subsection{The period distribution of $\delta$ Sct stars}

Chang et al. (2013) provide an overview of the observational properties of the then known $\delta$ Sct stars - their catalogue includes chemically peculiar $\delta$ Sct stars such as $\lambda$ Boo stars and roAp stars. The surveys which form the basis of this study have different depth and cadence to ours which will result in built-in biases. One example of this is the bimodal distribution of the $V$ mag of their sample which is due to the brighter objects being derived from the Hipparcos catalogue (Perryman et al. 1997, the product of the ESA's Space Astrometry Mission) and fainter objects from the MACHO (Alcock et al. 2000) and OGLE (Optical Gravitational Lensing Experiment, Woźniak et al. 2000) surveys.

OW has its own biases - for instance we have not attempted to include $\delta$ Sct stars with a pulsation period greater than $\sim 1 \mathrm{hr}$. In addition we have optical spectra of only 43 out of the 377 variables which we have identified as candidate $\delta$ Sct stars. In Paper III we determine the spectral type of these stars and find they have F spectral types which are consistent with that of $\delta$ Sct stars.

We highlight two questions here. What is the shortest period and what is the amplitude distribution of the $\delta$ Sct stars in our sample? The shortest period $\delta$ Sct stars in the Chang et al. (2013) catalogue have a period of $25.9 \mathrm{~min}$, although we note that the pre-main sequence $\delta$ Sct star HD 34282 (Amado et al. 2004) has a period of $18.1 \mathrm{~min}$. The shortest period $\delta$ Sct-like star we detect (OW J075633.0293226.6) has a period of 9.3 min which is shorter than those noted in Chang et al. (2013). Followup photometry and spectroscopy of this star is required to better characterise its properties.

Chang et al. (2013) adopt the same criteria for defining low amplitude $\delta$ Sct stars as Solano \& Fernley (1997), namely $\delta V=0.1 \mathrm{mag}$ with high amplitude $\delta$ Sct stars (HADS) having $\delta V>0.3$ mag. We show the percentage of stars in the Chang et al. (2013) catalogue which have an amplitude less than $0.01 \mathrm{mag}, 0.05 \mathrm{mag}$ and $0.10 \mathrm{mag}$ in Table 4. We do this for the whole sample of Chang et al. (2013) and also for the (much smaller) sample of stars in the range $14.5<V<20.5$ which is more comparable to OW. Whilst the Chang et al. (2013) sample shows many $\delta$ Sct stars with amplitudes greater than $0.1 \mathrm{mag}$, we find one star with an amplitude $>0.1 \mathrm{mag}$. It is possible that we are identifying very low amplitude variables of the sort observed using Kepler (e.g. Uytterhoeven et al. 2011). Bowman et al. (2016) find only two $\delta$ Sct stars out of 983 which were found to be HADS. (In Paper III we show that followup observations of our $\delta$ Sct sample indicate that very similar periods and amplitudes are observed at a second epoch of observation).

\begin{tabular}{|c|c|c|c|}
\hline $\begin{array}{l}\text { Range } \\
\text { (mag) }\end{array}$ & $\begin{array}{l}\text { Chang } \\
\text { all }\end{array}$ & $\begin{array}{r}\text { Chang } \\
(14.5<V<20.5)\end{array}$ & OW \\
\hline$\lesssim 0.01$ & $9.9 \%$ & $3.1 \%$ & $39.8 \%$ \\
\hline$\lesssim 0.05$ & $34.8 \%$ & $21.9 \%$ & $98.9 \%$ \\
\hline$\lesssim 0.10$ & $47.4 \%$ & $37.4 \%$ & $99.7 \%$ \\
\hline
\end{tabular}

Table 4. We compare the percentage of $\delta$ Sct stars with an amplitude less than three limits in three samples. We take all of the sample of Chang et al. (2013), the sample of Chang et al. in the $14.5<V<20.5$ range, and the OW sample which has been based on colour and period.

\section{CONCLUSIONS}

We have been able to identify a sample of blue stars which show variability in their light curve on a time-scale as short as $\sim 20 \mathrm{~min}$. Using an automated flagging procedure and subsequent manual inspection, we find that many of these stars are variable because diffraction spikes appear to rotate over the detector (and across the PSF of nearby stars) over the course of the $2 \mathrm{~h}$ observation as the telescope has an altaz mount. Although the fraction of contaminants is high, followup observations reported in Paper III show that our manual inspection is sufficiently robust that the sample of variable stars which pass this step are intrinsically variable on the time-scale identified in our pipeline. The underlying false positive rate is therefore very low. Our goal is to obtain high cadence light curves with a duration of $2 \mathrm{~h}$ over 400 square degrees close to the Galactic plane. As we expect $\mathrm{UCBs}$ to be rare, we will only be in a position to robustly determine the observed space density of short period UCBs once the survey approaches the target sky coverage.

\section{ACKNOWLEDGEMENTS}

The authors gratefully acknowledge funding from the Erasmus Mundus Programme SAPIENT, the National Research Foundation of South Africa (NRF), the Nederlandse Organisatie voor Wetenschappelijk Onderzoek (the Dutch Organization for Science Research), Radboud University and the University of Cape Town. The ESO observations used in this paper are based on observations made with ESO Telescopes at the La Silla Paranal Observatory under programme IDs: 088.D-4010(B), 090.D-0703(A), 090.D-0703(B), 091.D0716(A), 091.D-0716(B), 092.D-0853(B), 093.D-0937(A), 093.D-0753(A), 094.D-0502(A), 094.D-0502(B), and 177.D3023 (VPHAS+). This research has made use of the SIMBAD database, operated at CDS, Strasbourg, France. This research was made possible through the use of the AAVSO Photometric All-Sky Survey (APASS), funded by the Robert Martin Ayers Sciences Fund. Armagh Observatory is core funded by the Northern Ireland Government. This research has been facilitated by the NWO-NRF bilateral agreement got astronomical collaboration between The Netherlands and the Republic of South Africa. TRM acknowledges the support of STFC through a Consolidated Grant (ST/L000733). 


\section{REFERENCES}

Acker A., Chopinet M., Pottasch S. R., Stenholm B., 1987, A\&AS, 71,163

Alcock C., et al., 2000, ApJ, 542, 281

Amado P. J., Moya A., Suárez J. C., Martín-Ruíz S., Garrido R., Rodríguez E., Catala C., Goupil M.-J., 2004, IAUS, 224, 863

Anderson S. F., et al., 2005, AJ, 130, 2230

Anderson S. F., et al., 2008, AJ, 135, 2108

Bailey J., Cropper M. S., 1991, MNRAS, 253, 27

Barclay T., Ramsay G., Hakala P., Napiwotzki R., Nelemans G., Potter S., Todd I., 2011, MNRAS, 413, 2696

Blanton M. R., Roweis S., 2007, AJ, 133, 734

Bond H. E. 1979, in IAU Colloq. 53: White Dwarfs and Variable Degenerate Stars, ed. H. M. van Horn \& V. Weidemann, 266.

Bond H. E. 2000, in ASP Conf. Ser. 199: Asymmetrical Planetary Nebulae II: From Origins to Microstructures, 115

Bowman D. M., Kurtz D. W., Breger M., Murphy S. J., Holdsworth D. L., 2016, MNRAS, 460, 1970

Breedt E., et al., 2014, MNRAS, 443, 3174

Breger M., 2000, ASP Con Series, 210, 3

Britt C. T., et al., 2013, ApJ, 769, 120

Brown W. R., Kilic M., Hermes J. J., Allende Prieto C., Kenyon S. J., Winget D. E., 2011, ApJ, 737, L23

Brown W. R., Gianninas A., Kilic M., Kenyon S. J., Allende Prieto C., 2016, ApJ, 818, 155

Capaccioli M., Schipani P., 2011, Messenger, 146, 2

Carter P. J., et al., 2013, MNRAS, 429, 2143

Carter P. J., Steeghs D., Marsh T. R., Kupfer T., Copperwheat C. M., Groot P. J., Nelemans G., 2014a, MNRAS, 437, 2894

Carter P. J., et al., 2014b, MNRAS, 439, 2848

Chang S.-W., Protopapas P., Kim D.-W., Byun Y.-I., 2013, AJ, 145,132

Devor J., 2005, ApJ, 628, 411

Dilday B., et al., 2012, Sci, 337, 942

Drew J. E., et al., 2014, MNRAS, 440, 2036

Formiggini L., Leibowitz E. M., 2009, MNRAS, 396, 1507

Geier S., et al., 2013, A\&A, 554, 54

Gianninas A., Kilic M., Brown W. R., Canton P., Kenyon S. J., 2015, ApJ, 812, 167

Gilliland R. L., et al. 2010, ApJ, 713, 160

Graham M. J., Drake A. J., Djorgovski S. G., Mahabal A. A., Donalek C., Duan V., Maher A., 2013, MNRAS, 434, 3423

Hartman J. D., Gaudi B. S., Holman M. J., McLeod B. A., Stanek K. Z., Barranco J. A., Pinsonneault M. H., Kalirai J. S., 2008, ApJ, 675, 1254

Henden A. A., Levine S. E., Terrell D., Smith T. C., Welch D., 2012, JAVSO, 40, 430

Jonker P. J. et al., 2011, ApJS, 194, 18

Keller S. C., et al., 2007, PASA, 24, 1

Kuijken K., 2011, The Messenger, 146, 8

Lang D., Hogg D. W., Mierle K., Blanton M., Roweis S., 2010, AJ, 139, 1782

Kupfer T., et al., 2015, MNRAS, 453, 483

Law N. M., et al., 2009, PASP, 121, 1395

Levitan D., et al., 2011, ApJ, 739, 68

Levitan D., et al., 2013, MNRAS, 430, 996

Levitan D., et al., 2014, ApJ, 785, 114

Lomb, N.R. 1976, A\&SS, 39, 447

Macfarlane S. A., Toma R., Ramsay G., Groot P. J., Woudt P. A., Drew J. E., Barentsen G., Eislöffel J., 2015, MNRAS, 454, 507 (Paper I)

Mieske S., et al., 2013, The Messenger, 154, 12

Mikołajewska J., 2007, Baltic Astron., 16 , 1

Miszalski B., Acker A., Moffat A. F. J., Parker Q. A., Udalski A.,

2009, A\&A, 496, 813

Mróz P., et al., 2015, ApJS, 219, 26

Nelemans G., Steeghs D., Groot P. J, 2001, MNRAS, 326, 621
Nelemans G., Jonker P., 2010, NewAR, 54, 87

Nelemans G., 2013, ASPC, 467, 27, 9th LISA Symposium, Proceedings of the conference held 21-25 May 2012 at Bibliothque Nationale de France, Paris.

Nelemans G., Portegies Zwart S. F., Verbunt F., Yungelson L. R., 2001, A\&A, 368, 939

Nelemans G., Yungelson L. R., Portegies Zwart S. F., 2004, MNRAS, 349, 181

Perryman M. A. C. et al., 1997, A\&A, 323, 49

Press W.H., Teukolsky S.A., Vetterling W.T., Flannery B.P., 1992, Numerical Recipes in C, 2nd ed., New York, Cambridge University Press

Raddi R., et al., 2016, MNRAS, 457, 1988

Ramsay G., Hakala P., 2005, MNRAS, 360, 314

Ramsay G., Napiwotzki R., Hakala P., Lehto H., 2006, MNRAS, 371,957

Ramsay G. et al., 2014, MNRAS, 437, 132

Roelofs G. H. A., Groot P. J., Marsh T. R., Steeghs D., Barros S. C. C., Nelemans G., 2005, MNRAS, 361, 487

Roelofs G. H. A., Nelemans G., Groot P. J., 2007, MNRAS, 382, 685

Roelofs G. H. A., Rau A., Marsh T. R., Steeghs D., Groot P. J., Nelemans G., 2010, ApJ, 711, L138

Scargle J.D., 1982, ApJ, 263, 835

Schipani P., et al., 2012, SPIE, 8444E, 1C

Schwarzenberg-Czerny A., 1989, MNRAS, 241, 153

Sokoloski J. L., Bildsten L., 1999, ApJ, 517, 919

Solano E., Fernley J., 1997, A\&AS, 122, 131

Solheim J.-E., 2000, PASP, 122, 1133

Tamuz O., Mazeh T., Zucker S., 2005, MNRAS, 356, 1466

Wozniak P. R., 2000, AcA, 50, 421

Uytterhoeven K. et al., 2011, A\&A, 534, A125

Woudt P. A., Warner B., Spark M., 2005, MNRAS, 364, 107

Woźniak, P. R., et al., 2000, ApJ, 540, 65

Verbeek K., et al., 2012, MNRAS, 420, 1115

Verbeek K., et al., 2013, MNRAS, 434, 2727

York D. G., 2000, AJ, 120, 1579

Yungelson et al. 1995, ApJ, 447, 656

Zechmeister M., Kürster M., 2009, A\&A, 496, 577

Zorotovic M., Schreiber M. R., Parsons S. G., Gänsicke B. T.,

Hardy A., Agurto-Gangas C., Nebot Gómez-Morán A., Rebassa-

Mansergas A., Schwope A. D., 2016, MNRAS, 457, 3867

\section{APPENDIX A: TABLES}


Table A1. The observation log of field pointings made over ESO Semester 90-94, where we show the sky co-ordinates in equatorial and Galactic co-ordinates; the calendar date of the start of the observations and the range in the seeing in arcsec.

\begin{tabular}{|c|c|c|c|c|c|c|}
\hline Field & $\begin{array}{c}\text { RA } \\
(\mathrm{J} 2000)\end{array}$ & $\begin{array}{c}\text { DEC } \\
(\mathrm{J} 2000)\end{array}$ & $l$ & $b$ & $\begin{array}{c}\text { Date } \\
\text { (dd-mm-yy) }\end{array}$ & $\begin{array}{c}\text { Seeing }\left({ }^{\prime \prime}\right) \\
\text { Mean, } \sigma\end{array}$ \\
\hline \multicolumn{7}{|c|}{ Semester P90 } \\
\hline $29 \mathrm{a}$ & $07: 48: 15.9$ & $-29: 39: 20.1$ & 245.48 & -2.05 & $07-12-2012$ & 0.790 .43 \\
\hline $29 \mathrm{~b}$ & $07: 52: 52.1$ & $-29: 39: 15.9$ & 245.98 & -1.19 & 07-12-2012 & 0.790 .43 \\
\hline $31 \mathrm{a}$ & $07: 29: 34.1$ & $-29: 41: 07.4$ & 243.52 & -5.61 & 04-03-2013 & 0.790 .43 \\
\hline $31 b$ & $07: 34: 10.3$ & $-29: 41: 02.6$ & 243.99 & -4.73 & 04-03-2013 & $\begin{array}{lll}0.80 & 0.44\end{array}$ \\
\hline \multicolumn{7}{|c|}{ Semester P91 } \\
\hline $6 \mathrm{a}$ & $17: 02: 19.1$ & $-27: 59: 57.3$ & 355.42 & 8.40 & 01-08-2013 & 1.190 .55 \\
\hline $6 \mathrm{~b}$ & $17: 06: 50.8$ & $-28: 00: 02.7$ & 356.02 & 7.60 & 01-08-2013 & 1.170 .54 \\
\hline $12 \mathrm{a}$ & $07: 06: 21.4$ & $-30: 00: 02.6$ & 241.51 & -10.25 & 06-04-2013 & 0.850 .45 \\
\hline $12 \mathrm{~b}$ & $07: 10: 58.5$ & $-29: 59: 57.4$ & 241.95 & -9.34 & 06-04-2013 & 0.900 .47 \\
\hline $13 \mathrm{a}$ & $17: 15: 43.4$ & $-29: 59: 57.2$ & 355.54 & 4.85 & $16-05-2013$ & 1.260 .54 \\
\hline $13 b$ & $17: 20: 20.5$ & $-30: 00: 02.8$ & 356.11 & 4.04 & 16-05-2013 & 1.290 .54 \\
\hline $15 \mathrm{a}$ & $17: 46: 37.6$ & $-23: 59: 57.3$ & 4.34 & 2.37 & 01-06-2013 & 1.170 .52 \\
\hline $15 \mathrm{~b}$ & $17: 51: 00.3$ & $-24: 00: 02.7$ & 4.85 & 1.52 & 01-06-2013 & 1.170 .52 \\
\hline $17 \mathrm{a}$ & $17: 37: 51.6$ & $-24: 59: 57.3$ & 2.43 & 3.54 & 30-06-2013 & 1.100 .52 \\
\hline $17 \mathrm{~b}$ & $17: 42: 16.3$ & $-25: 00: 02.7$ & 2.96 & 2.70 & 30-06-2013 & 1.100 .52 \\
\hline $18 \mathrm{a}$ & $17: 46: 47.6$ & $-24: 59: 57.3$ & 3.50 & 1.82 & 15-07-2013 & 1.010 .48 \\
\hline $18 \mathrm{~b}$ & $17: 51: 12.3$ & $-25: 00: 02.7$ & 4.01 & 0.97 & 15-07-2013 & 0.980 .47 \\
\hline $20 \mathrm{a}$ & $18: 07: 57.6$ & $-24: 59: 57.3$ & 5.90 & -2.33 & 01-08-2013 & 1.450 .62 \\
\hline $20 \mathrm{~b}$ & $18: 12: 22.3$ & $-25: 00: 02.7$ & 6.38 & -3.20 & 01-08-2013 & 1.420 .61 \\
\hline $21 \mathrm{a}$ & $18: 16: 54.6$ & $-24: 59: 57.3$ & 6.87 & -4.11 & 01-07-2013 & 1.540 .71 \\
\hline $21 b$ & $18: 21: 19.3$ & $-25: 00: 02.7$ & 7.34 & -4.99 & 01-07-2013 & 1.500 .70 \\
\hline $23 a$ & $17: 37: 56.5$ & $-25: 59: 57.3$ & 1.60 & 3.00 & 08-08-2013 & 1.070 .50 \\
\hline $23 b$ & $17: 42: 23.4$ & $-26: 00: 02.7$ & 2.13 & 2.15 & 08-08-2013 & 1.070 .50 \\
\hline $24 \mathrm{a}$ & $17: 49: 26.5$ & $-25: 59: 57.3$ & 2.95 & 0.79 & 11-08-2013 & 1.020 .49 \\
\hline $24 \mathrm{~b}$ & $17: 53: 53.4$ & $-26: 00: 02.7$ & 3.46 & -0.07 & 11-08-2013 & 1.000 .48 \\
\hline $29 a$ & $17: 40: 03.3$ & $-26: 59: 57.2$ & 1.00 & 2.06 & $10-07-2013$ & 1.240 .53 \\
\hline $29 \mathrm{~b}$ & $17: 44: 32.6$ & $-27: 00: 02.8$ & 1.53 & 1.21 & 10-07-2013 & 1.260 .53 \\
\hline $31 \mathrm{a}$ & $17: 58: 05.3$ & $-26: 59: 57.2$ & 3.07 & -1.38 & $31-07-2013$ & 0.960 .47 \\
\hline $31 b$ & $18: 02: 34.6$ & $-27: 00: 02.8$ & 3.57 & -2.25 & 31-07-2013 & 0.910 .46 \\
\hline $32 \mathrm{a}$ & $18: 07: 16.3$ & $-26: 59: 57.2$ & 4.08 & -3.16 & 06-08-2013 & 1.150 .55 \\
\hline $32 \mathrm{~b}$ & $18: 11: 45.6$ & $-27: 00: 02.8$ & 4.56 & -4.04 & 06-08-2013 & 1.150 .55 \\
\hline \multicolumn{7}{|c|}{ Semester P92 } \\
\hline $32 \mathrm{a}$ & $07: 06: 39.0$ & $-27: 40: 57.3$ & 239.42 & -9.18 & $26-12-2013$ & 1.140 .53 \\
\hline $32 b$ & 07:11:10.0 & $-27: 40: 52.2$ & 239.86 & -8.28 & 26-12-2013 & 1.080 .52 \\
\hline $36 a$ & $08: 24: 25.4$ & $-27: 40: 56.9$ & 248.08 & 5.69 & 24-01-2014 & 1.320 .55 \\
\hline $36 \mathrm{~b}$ & $08: 28: 56.3$ & $-27: 40: 52.6$ & 248.65 & 6.51 & 24-01-2014 & 1.330 .55 \\
\hline $37 \mathrm{a}$ & $08: 33: 37.2$ & $-27: 40: 56.8$ & 249.26 & 7.36 & 24-01-2014 & 0.720 .40 \\
\hline $37 \mathrm{~b}$ & 08:38:08.2 & $-27: 40: 52.7$ & 249.86 & 8.16 & 24-01-2014 & 0.730 .40 \\
\hline $46 \mathrm{a}$ & $08: 25: 23.3$ & $-29: 41: 07.2$ & 249.85 & 4.72 & 02-03-2014 & 1.030 .50 \\
\hline $46 \mathrm{~b}$ & $08: 29: 59.5$ & $-29: 41: 02.8$ & 250.43 & 5.54 & 02-03-2014 & 1.040 .50 \\
\hline \multicolumn{7}{|c|}{ Semester P93 } \\
\hline $94 \mathrm{a}$ & $18: 11: 24.0$ & $-27: 59: 57.1$ & 272.85 & -28.00 & 10-04-2014 & 1.080 .49 \\
\hline $94 \mathrm{~b}$ & $18: 15: 55.8$ & $-28: 00: 02.9$ & 273.98 & -28.00 & 10-04-2014 & 1.090 .50 \\
\hline $47 \mathrm{a}$ & $17: 11: 34.4$ & $-31: 59: 57.0$ & 257.89 & -32.00 & $24-06-2014$ & 0.810 .44 \\
\hline $47 \mathrm{~b}$ & $17: 16: 17.4$ & $-32: 00: 03.0$ & 259.07 & -32.00 & 24-06-2014 & 0.840 .44 \\
\hline $53 a$ & $17: 11: 28.0$ & $-27: 59: 57.1$ & 257.86 & -28.00 & 25-08-2014 & 1.170 .56 \\
\hline $53 \mathrm{~b}$ & $17: 15: 59.8$ & $-28: 00: 02.9$ & 258.99 & -28.00 & $25-08-2014$ & 1.160 .55 \\
\hline $83 a$ & $17: 46: 55.5$ & $-21: 59: 57.2$ & 266.73 & -22.00 & 20-06-2014 & 1.070 .50 \\
\hline $83 b$ & $17: 51: 14.3$ & $-22: 00: 02.8$ & 267.80 & -22.00 & 20-06-2014 & 1.060 .49 \\
\hline $84 a$ & $17: 55: 52.5$ & $-21: 59: 57.2$ & 268.96 & -22.00 & 23-06-2014 & 0.970 .53 \\
\hline $84 \mathrm{~b}$ & 18:00:11.3 & $-22: 00: 02.8$ & 270.04 & -22.00 & 23-06-2014 & 0.950 .52 \\
\hline $85 \mathrm{a}$ & $18: 04: 30.5$ & $-21: 59: 57.2$ & 271.12 & -22.00 & 24-06-2014 & 1.150 .52 \\
\hline $85 \mathrm{~b}$ & 18:08:49.3 & $-22: 00: 02.8$ & 272.20 & -22.00 & 24-06-2014 & 1.150 .54 \\
\hline
\end{tabular}


Table A1. Continued ....

\begin{tabular}{|c|c|c|c|c|c|c|}
\hline Field & $\begin{array}{c}\text { RA } \\
(\mathrm{J} 2000)\end{array}$ & $\begin{array}{c}\text { DEC } \\
(\mathrm{J} 2000)\end{array}$ & $l$ & $b$ & $\begin{array}{c}\text { Date } \\
\text { (dd-mm-yy) }\end{array}$ & $\begin{array}{c}\text { seeing }\left({ }^{\prime \prime}\right) \\
\text { Mean, } \sigma\end{array}$ \\
\hline \multicolumn{7}{|c|}{ Semester P93 (Cont) } \\
\hline $86 \mathrm{a}$ & $18: 13: 07.5$ & $-21: 59: 57.2$ & 273.28 & -22.00 & 01-08-2014 & 1.110 .50 \\
\hline $86 \mathrm{~b}$ & $18: 17: 26.3$ & $-22: 00: 02.8$ & 274.35 & -22.00 & $01-08-2014$ & 1.120 .51 \\
\hline $90 \mathrm{a}$ & $17: 53: 32.6$ & $-22: 59: 57.2$ & 268.38 & -23.00 & $30-07-2014$ & $0.97 \quad 0.47$ \\
\hline $90 \mathrm{~b}$ & $17: 57: 53.2$ & $-23: 00: 02.8$ & 269.47 & -23.00 & $30-07-2014$ & 0.980 .47 \\
\hline $91 \mathrm{a}$ & 18:06:01.6 & $-22: 59: 57.2$ & 271.50 & -23.00 & $30-07-2014$ & $\begin{array}{lll}0.81 & 0.43\end{array}$ \\
\hline $91 \mathrm{~b}$ & $18: 10: 22.2$ & $-23: 00: 02.8$ & 272.59 & -23.00 & $30-07-2014$ & $\begin{array}{lll}0.81 & 0.43\end{array}$ \\
\hline $51 \mathrm{a}$ & $17: 19: 17.3$ & $-26: 59: 57.1$ & 259.82 & -27.00 & 01-07-2014 & 1.290 .55 \\
\hline $51 \mathrm{~b}$ & $17: 23: 46.6$ & $-27: 00: 03.0$ & 260.94 & -27.00 & 01-07-2014 & $\begin{array}{lll}1.31 & 0.56\end{array}$ \\
\hline $97 \mathrm{a}$ & $17: 16: 50.0$ & $-30: 59: 57.0$ & 259.20 & -31.00 & $27-05-2014$ & 1.180 .54 \\
\hline $97 \mathrm{~b}$ & $17: 21: 29.9$ & $-31: 00: 03.0$ & 260.37 & -31.00 & $27-05-2014$ & 1.150 .52 \\
\hline $99 a$ & $17: 35: 27.0$ & $-30: 59: 56.9$ & 263.86 & -31.00 & $23-06-2014$ & 0.910 .45 \\
\hline $99 \mathrm{~b}$ & $17: 40: 06.9$ & $-31: 00: 03.1$ & 265.02 & -31.00 & $23-06-2014$ & 0.890 .45 \\
\hline $100 \mathrm{a}$ & $17: 44: 49.0$ & $-30: 59: 56.9$ & 266.20 & -31.00 & $27-07-2014$ & 0.880 .47 \\
\hline $100 \mathrm{~b}$ & $17: 49: 28.9$ & $-31: 00: 03.1$ & 267.37 & -31.00 & $27-07-2014$ & 0.890 .48 \\
\hline $101 \mathrm{a}$ & $17: 54: 17.0$ & $-30: 59: 56.9$ & 268.57 & -31.00 & 29-07-2014 & $\begin{array}{lll}1.12 & 0.51\end{array}$ \\
\hline $101 \mathrm{~b}$ & $17: 58: 56.9$ & $-31: 00: 03.1$ & 269.73 & -31.00 & $29-07-2014$ & 1.010 .51 \\
\hline $102 \mathrm{a}$ & $18: 03: 40.0$ & $-30: 59: 56.9$ & 270.91 & -31.00 & $31-07-2014$ & 1.210 .61 \\
\hline $102 \mathrm{~b}$ & $18: 08: 19.9$ & $-31: 00: 03.1$ & 272.08 & -31.00 & $31-07-2014$ & 1.250 .63 \\
\hline $48 \mathrm{a}$ & $17: 21: 08.5$ & $-31: 59: 56.9$ & 260.28 & -32.00 & $23-06-2014$ & 1.260 .56 \\
\hline $48 \mathrm{~b}$ & $17: 25: 51.4$ & $-32: 00: 03.1$ & 261.46 & -32.00 & 23-06-2014 & 1.240 .58 \\
\hline $103 a$ & $17: 30: 59.5$ & $-31: 59: 56.9$ & 262.74 & -32.00 & 01-08-2014 & 0.980 .49 \\
\hline 103b & $17: 35: 42.4$ & $-32: 00: 03.1$ & 263.92 & -32.00 & 01-08-2014 & 0.990 .49 \\
\hline \multicolumn{7}{|c|}{ Semester P94 } \\
\hline $34 a$ & $07: 29: 25.3$ & $-27: 40: 57.4$ & 112.35 & -27.68 & $14-01-2015$ & 0.950 .47 \\
\hline $34 \mathrm{~b}$ & $07: 33: 56.2$ & $-27: 40: 52.1$ & 113.48 & -27.68 & $14-01-2015$ & 0.930 .46 \\
\hline $39 a$ & $07: 49: 12.9$ & $-31: 40: 46.6$ & 117.30 & -31.67 & $17-12-2014$ & $\begin{array}{lll}0.89 & 0.47\end{array}$ \\
\hline 39b & $07: 53: 54.9$ & $-31: 40: 41.4$ & 118.47 & -31.67 & $17-12-2014$ & $0.87 \quad 0.44$ \\
\hline $113 a$ & $07: 48: 14.4$ & $-30: 40: 46.6$ & 117.06 & -30.67 & $16-02-2015$ & 0.790 .43 \\
\hline $113 \mathrm{~b}$ & $07: 52: 53.4$ & $-30: 40: 41.4$ & 118.22 & -30.67 & $16-02-2015$ & $\begin{array}{lll}0.77 & 0.41\end{array}$ \\
\hline $24 a$ & $07: 22: 15.0$ & $-25: 40: 49.6$ & 110.56 & -25.68 & $24-02-2015$ & $0.83 \quad 0.44$ \\
\hline $24 b$ & $07: 26: 41.3$ & $-25: 40: 44.3$ & 111.67 & -25.67 & $24-02-2015$ & 0.840 .44 \\
\hline $40 \mathrm{a}$ & $07: 58: 12.9$ & $-31: 40: 46.5$ & 119.55 & -31.67 & $27-12-2014$ & 0.870 .46 \\
\hline $40 \mathrm{~b}$ & 08:02:54.9 & $-31: 40: 41.5$ & 120.72 & -31.67 & $27-12-2014$ & $\begin{array}{lll}0.88 & 0.46\end{array}$ \\
\hline $107 \mathrm{a}$ & $07: 22: 21.1$ & $-24: 30: 23.6$ & 110.58 & -24.50 & 23-01-2015 & 1.170 .54 \\
\hline $107 \mathrm{~b}$ & $07: 26: 44.8$ & $-24: 30: 18.4$ & 111.68 & -24.50 & 23-01-2015 & 1.190 .56 \\
\hline $108 \mathrm{a}$ & $07: 31: 21.1$ & $-24: 30: 23.6$ & 112.83 & -24.50 & $26-01-2015$ & 1.140 .53 \\
\hline $108 \mathrm{~b}$ & $07: 35: 44.8$ & $-24: 30: 18.4$ & 113.93 & -24.50 & $26-01-2015$ & 1.160 .52 \\
\hline $38 \mathrm{a}$ & $07: 18: 52.1$ & $-23: 30: 46.6$ & 109.71 & -23.51 & $13-01-2015$ & 0.780 .43 \\
\hline $38 \mathrm{~b}$ & $07: 23: 13.7$ & $-23: 30: 41.4$ & 110.80 & -23.51 & 13-01-2015 & $0.78 \quad 0.43$ \\
\hline $42 \mathrm{a}$ & $08: 16: 12.9$ & $-31: 40: 46.5$ & 124.05 & -31.67 & $24-02-2015$ & 0.870 .46 \\
\hline $42 \mathrm{~b}$ & $08: 20: 54.9$ & $-31: 40: 41.6$ & 125.22 & -31.67 & $24-02-2015$ & 0.870 .46 \\
\hline $115 \mathrm{a}$ & 08:12:07.6 & $-30: 40: 46.5$ & 123.03 & -30.67 & 13-03-2015 & 0.960 .48 \\
\hline 115b & $08: 16: 46.6$ & $-30: 40: 41.6$ & 124.19 & -30.67 & $13-03-2015$ & $\begin{array}{lll}0.95 & 0.47\end{array}$ \\
\hline $116 a$ & $08: 21: 21.7$ & $-30: 40: 46.3$ & 125.34 & -30.67 & $22-01-2015$ & $\begin{array}{lll}1.09 & 0.54\end{array}$ \\
\hline $116 \mathrm{~b}$ & $08: 26: 00.7$ & $-30: 40: 41.7$ & 126.50 & -30.67 & $22-01-2015$ & 1.060 .52 \\
\hline $25 \mathrm{a}$ & $07: 31: 12.5$ & $-25: 40: 49.5$ & 112.80 & -25.68 & 22-03-2015 & 1.050 .50 \\
\hline $25 \mathrm{~b}$ & $07: 35: 38.7$ & $-25: 40: 44.1$ & 113.91 & -25.67 & $22-03-2015$ & 1.040 .50 \\
\hline $33 a$ & $07: 36: 37.5$ & $-23: 30: 46.5$ & 114.15 & -23.51 & 13-01-2015 & $\begin{array}{lll}0.86 & 0.45\end{array}$ \\
\hline $33 \mathrm{~b}$ & $07: 40: 59.1$ & $-23: 30: 41.5$ & 115.24 & -23.51 & $13-01-2015$ & 0.870 .45 \\
\hline $36 \mathrm{a}$ & 08:07:12.9 & $-31: 40: 46.5$ & 121.80 & -31.67 & $23-02-2015$ & $0.90 \quad 0.45$ \\
\hline $36 \mathrm{~b}$ & $08: 11: 54.9$ & $-31: 40: 41.5$ & 122.97 & -31.67 & 23-02-2015 & $0.92 \quad 0.46$ \\
\hline $112 \mathrm{a}$ & $07: 37: 56.4$ & $-30: 40: 46.6$ & 114.48 & -30.67 & $14-01-2015$ & 0.890 .45 \\
\hline $112 \mathrm{~b}$ & $07: 42: 35.4$ & $-30: 40: 41.4$ & 115.64 & -30.67 & $14-01-2015$ & $\begin{array}{lll}0.88 & 0.45\end{array}$ \\
\hline $117 \mathrm{a}$ & $07: 39: 54.9$ & $-31: 40: 46.7$ & 114.97 & -31.67 & 24-01-2015 & 0.990 .47 \\
\hline $117 \mathrm{~b}$ & $07: 44: 36.9$ & $-31: 40: 41.3$ & 116.15 & -31.67 & 24-01-2015 & $\begin{array}{lll}1.00 & 0.47\end{array}$ \\
\hline $109 a$ & $07: 39: 56.5$ & $-24: 30: 46.5$ & 114.98 & -24.51 & $23-02-2015$ & $0.92 \quad 0.46$ \\
\hline $109 \mathrm{~b}$ & $07: 44: 20.2$ & $-24: 30: 41.5$ & 116.08 & -24.51 & 23-02-2015 & 0.940 .47 \\
\hline $114 a$ & $08: 02: 50.4$ & $-30: 40: 46.5$ & 120.71 & -30.67 & $25-02-2015$ & 0.990 .49 \\
\hline $114 \mathrm{~b}$ & $08: 07: 29.4$ & $-30: 40: 41.5$ & 121.87 & -30.67 & $25-02-2015$ & 1.000 .50 \\
\hline
\end{tabular}



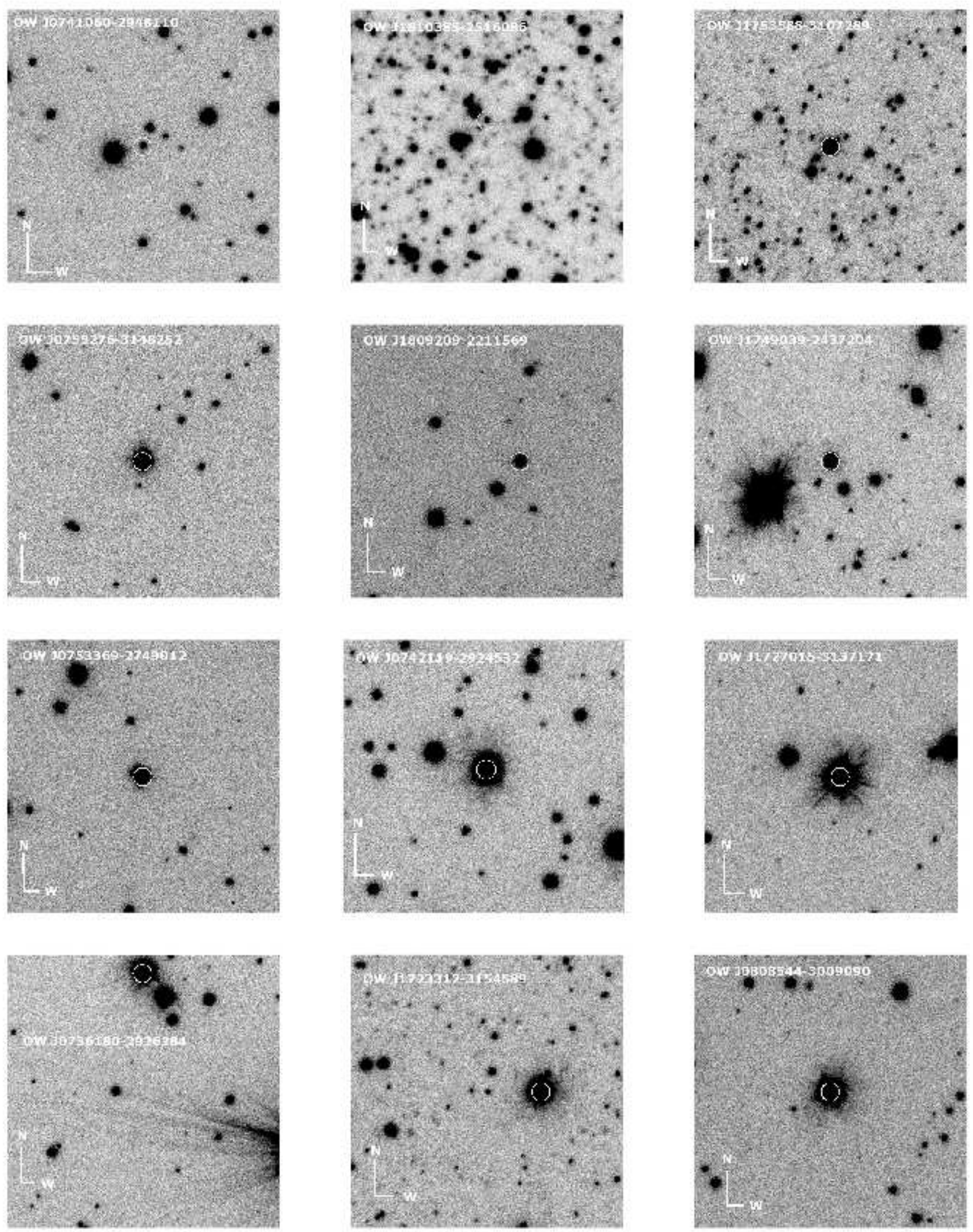

Figure Al: Finding Charts for the variable stars outlined in Table 3. The field-of-view in each chart is $1^{\prime} \times 1^{\prime}$ across. 

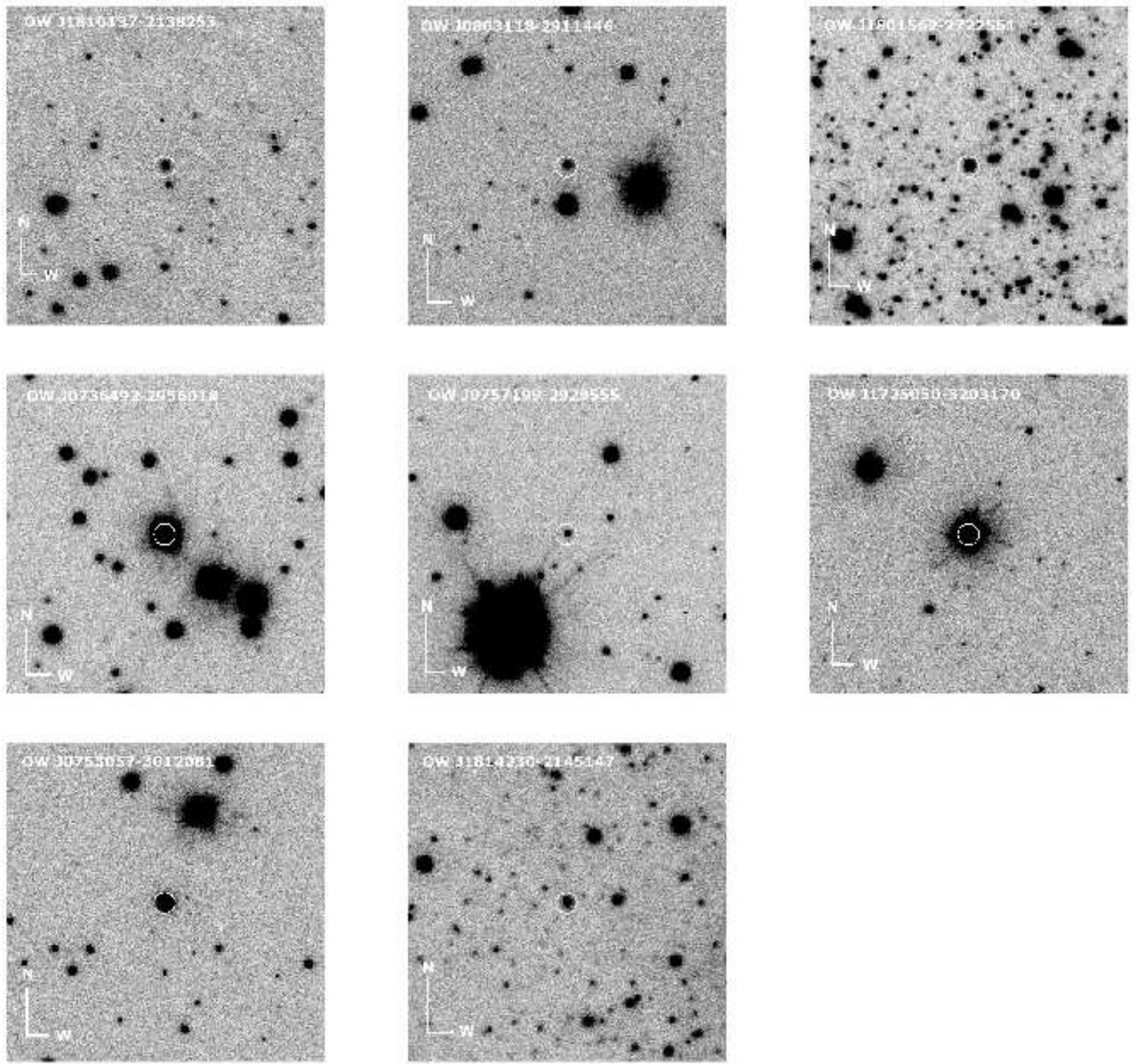

Figure Al: (cont). We note that although OW J0757199-2929555 appears close to a diffraction spike, manual verification confirms the period of $98.7 \mathrm{~m}$ and amplitude of 0.44 mag. 